4

\title{
Caenorhabditis elegans locomotion is affected by internalized paramagnetic nanoparticles in the presence of magnetic field
}

Mechanical Engineering, University of Michigan, Ann Arbor, Michigan

${ }^{2}$ Internal Medicine, Division of Geriatrics and Palliative Medicine, Medical School, University of Michigan, Ann Arbor, Michigan

${ }^{3}$ Electrical Engineering and Computer Science, University of Michigan, Ann Arbor, Michigan

${ }^{4}$ University of Michigan and Shanghai Jiao Tong University Joint Institution, Shanghai, China

* present address: Mechanical Engineering, University of Michigan, Ann Arbor, Michigan

** present address: Robotics System Development, Carnegie Mellon University, Pittsburg,

Pennsylvania

*** present address: Biomedical Engineering, University of Michigan, Ann Arbor, Michigan

+ corresponding author: Bogdan Epureanu

Email: epureanu@umich.edu

Running Title: nanoparticles C. elegans locomotion

Keywords: C. elegans; nanoparticles; magnetic field gradient; locomotion; paramagnetic

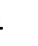




\section{Summary Statement}

C. elegans with internalized paramagnetic nanoparticles are placed inside magnetic field to explore effects on locomotion. Results support the potential of $C$. elegans to investigate the impact of the above environmental factors on behavior.

\section{Abstract}

C. elegans nematodes are a model organism used broadly to investigate the impact of environmental factors on physiology and behavior. Here, C. elegans with internalized paramagnetic nanoparticles were placed inside magnetic field to explore effects on locomotion. We hypothesize that internalized paramagnetic nanoparticles combined with external magnetic field affect $C$. elegans' locomotion machinery. To test our hypothesis, we used young adult $C$. elegans fed on bacteria mixed with paramagnetic nanoparticles of $1 \mu \mathrm{m}, 100 \mathrm{~nm}$ and $40 \mathrm{~nm}$ diameter. The presence of nanoparticles inside the worms' body (alimentary canal, body muscle) was verified by fluorescent and electron microscopy. A custom-made software was used to track freely moving $C$. elegans in the absence or presence of magnetic field sequentially for $200+200$ sec. We used established metrics to quantify locomotion-related parameters, including posture, motion and path features. Key features of $C$. elegans locomotion (increased body bends and stay ratio, decreased range, forward movement, and speed along the magnetic field) were affected in worms with internalized nanoparticles of $100 \mathrm{~nm}$ and $1 \mu \mathrm{m}$ in the presence of magnetic field, in contrast to untreated worms. Our work contributes on clarifying the effect of internalized paramagnetic nanoparticles, combined with magnetic field, on C. elegans locomotion.

\section{Introduction}

The effects of magnetic field (MF) on living organisms have been a target of numerous research efforts, with their number increasing significantly during the last decades (Ghodbane et al., 2013; Hong, 1995; Shaw et al., 2015). In addition to the interest scientific community shows on the effect of alternating MF on cells (Ueno et al., 1986; Öcal, 2008; Belova and Acosta-Avalos, 2015), static MF effects have gained attention also (Miyakoshi, 2005; Teodori et al., 2002) mainly due to their correlation with activity linked to the modern way of living (Lewczuk, 2014). The type 
framework for the possible impact of high-gradient MF of various sources on cells' molecular components and function (Zablotskii et al., 2016).

Model organisms have been a successful resource to study MF effects on various types of cells and tissues (Osipova et al., 2016; Shcherbakov et al.; Malkemper et al., 2015; Kumari et al., 2017). Invertebrate models, like Drosophila melanogaster, have been used since the 80's (Fedele et al., 2014; Naito et al., 2012; Ramirez et al., 1983; Kale and Baum, 1980; Bae et al., 2016; Giachello et al., 2016). Interestingly, even though the nematode C. elegans has been an emblematic model organism to study the impact of a plethora of stimuli and environmental factors on behavior and physiology (Cheung et al., 2005; De Bono and Bargmann, 1998; Liedtke et al., 2003; Hedgecock and Russell, 1975; Ward et al., 2008), only recently it has been used in MF related work (Vidal-Gadea, 2015; Njus, 2015; Long et al., 2015; Wang et al., 2015; Lee et al., 2010), in which the first animal magnetosensory neurons were identified (Vidal-Gadea, 2015). The presence of biogenic magnetite has also been reported in C. elegans (Cranfield et al., 2004).

Nanoparticles uptake by $C$. elegans worms has been a successful means to evaluate toxicity of heavy metals and pollutants (Khare et al., 2011; Meyer et al., 2010; Kim et al., 2017), and the importance of $C$. elegans as a model system for in vivo nanoparticle assessment has been specifically highlighted (Gonzalez-Moragas et al., 2015a). Worms' behavior (Ma et al., 2009) and locomotion ( $\mathrm{Li}$ et al., 2012; Wu et al., 2012) have been evaluated under the influence of internalized metal nanoparticles. In addition, magnetized nanoparticles have been used to activate ion channels in C. elegans through heating (Huang et al., 2010). However, only very recently internalized nanoparticles were used to locally enhance MF in the worms' body and study the subsequent impact on its metabolism (Wang et al., 2017).

C. elegans locomotion has been a major behavioral output used to investigate the impact of genetic background, environmental factors and diverse treatments on the worm's nervous system (Gourgou, 2016; Li et al., 2016; Liu, 2013; Hsu et al., 2009; Parida et al., 2014; PierceShimomura et al., 2008). Locomotion features have been characterized and quantified extensively and are being used as an indicator of $C$. elegans physiological status and healthspan (Bansal et al., 2015; Shtonda and Avery, 2006; Peliti et al., 2013; Hahm et al., 2015). Therefore, locomotion is one of the first behaviors investigated to define whether $C$. elegans nematodes are sensitive to an environmental factor of interest.

The abovementioned scientific premises, the remaining need to clarify MF effects on animal physiology, and the increasing interest in the sensitivity of $C$. elegans to MF, indicate that the investigation of MF gradient effects on worms' behavior comes at a mature point. We hypothesize that internalized paramagnetic nanoparticles combined with external magnetic field 
102

103

104

105

106

107

108

109

110

111

112

113

114

115

116

117

118

119

120

121

122

123

124

125

126

127

128

129

130

131

132

133

134

135

affect $C$. elegans' locomotive behavior. To test our hypothesis, we used internalized paramagnetic nanoparticles that can generate an effect inside the worms' body, in the closest possible proximity with tissues and cells. We used locomotion as a quantifiable and revealing behavioral expression to determine the effect of MF combined with the internalized nanoparticles. Our results demonstrate a response of $C$. elegans locomotion machinery to internalized paramagnetic nanoparticles in combination with MF gradients, and they pave the way for future studies seeking to clarify the participation of excitable cells, muscles and potentially even neurons, to this still uncharacterized behavior.

\section{Results}

\section{Magnetic field gradient characterization}

The simulation results for both electromagnets agreed with the experimental results provided by the manufacturer (Fig. S1). An overview of the MF around the electromagnets and the geometry of the model in COMSOL are presented in Figure 2A and a clear view of the MF on the plane of the worm plate surface is presented in Figure 2B. The arrows demonstrate the direction of the MF between the two electromagnets. The contours show that the MF is stronger near the electromagnets, as expected.

We focused on the features of the MF and the forces generated on the plate surface, where the worms' locomotion takes place. The MF was almost one-dimensional on the plate surface and was stronger nearer the electromagnets (Fig. $2 \mathrm{C}$ ). There were 9 components for the gradient of the MF. In Figure 2D, the strongest component of the MF gradient is shown (Bxx), which is parallel to $x$ axis. The magnitude of the gradient was larger near the electromagnets.

The nanoparticles create secondary MFs in the presence of an external MF. Details on calculating the forces that were created by the particles used in the present study can be found in the Supplementary Information section. The magnitude of the MF flux was calculated in MATLAB using Eqs. [2] and [3] of Supplementary Information for configurations along the $x$ and $y$ axes of three nanoparticles (as shown in Fig. 3A-3D). The MF was stronger close to the particles for both configurations and decayed rapidly as the distance from the particles increased (Fig. 3E). The force between the particles in the $x$ direction was attractive, while the force between the particles in the $y$ direction was repulsive. The attractive forces between the particles allow them to form chain-like structures, if they are not interrupted by the medium in which the particles are located (Mirzakhalili et al., 2017; Nakata et al., 2008). The magnetic moment of the external MF for the particles on the worm plate surface is depicted in Figure 3F. 


\section{Confirmation of nanoparticles uptake and particle localization in C. elegans' body}

Nanoparticles mixed with bacterial food were successfully internalized, as verified by microscopy methods, selected according to the properties of each particle group (Fig. 4). The presence of $1 \mu \mathrm{m}$ paramagnetic particles (Table 1) in the worm's intestine and in the pharynx around the grinder area was verified by bright field microscopy. The particles appear as dark (copper-colored) objects accumulated in the alimentary canal (Fig. 4A, right panel), whereas control animals' intestine area appears transparent (Fig. 4A, left panel). Uptake of $100 \mathrm{~nm}$ fluorescent, paramagnetic particles (Table 1) was verified by fluorescent microscopy. The particles appear to accumulate along the intestine lumen and in the pharynx, as shown when filters for rhodamine, the fluorescent substance with which the particles were coated (see Table 1 for particles properties), were used (Fig. 4B). Successful feeding on $40 \mathrm{~nm}$ paramagnetic particles (Table 1) was confirmed by scanning electron microscopy. As shown in Figure 4C, when using the circular backscatter (CBS) detector, $40 \mathrm{~nm}$ particles were visualized as white dots under the worm cuticle, in the broad area downstream of the pharynx and along the alimentary canal. The deformation of the sample due to the process followed allowed for obtaining only the approximate location of the particles. The white dots which represent the particles appear in different sizes, which might be attributed to particle aggregates or to the different depth at which the particles were located.

To investigate the particles localization in the worms' body, and to decipher whether they pass the intestine barrier, we used transmission electron microscopy. TEM images show that the particles can be found in the intestine (Fig. 5D) and the intestine lumen (Fig. 5E). Interestingly, particles aggregates were also detected inside muscle tissue, very close to the body wall (Fig. $5 \mathrm{~F})$. Therefore, the nanoparticles can be located very close to excitable cells.

\section{Analysis of C. elegans locomotion}

Analysis of selected locomotion features revealed that worms fed with $1 \mu \mathrm{m}$ and $100 \mathrm{~nm}$ diameter paramagnetic nanoparticles, when they moved freely in MF, had altered locomotion dynamics, compared to worms without internalized nanoparticles.

We examined selected posture features for each worm of each group, namely the total body bends in degrees, and the number of bends (bend count) realized per worm. The total body bends were not affected by the presence of either particles or of MF (Fig. 6A). However, there was a significant increase in the number of bends per worm of Group 100 (Fig. 6B, WSR test $p$ value $=0.031)$ when the MF was on. 
Next, we analyzed features related to the motion state and velocity of the worms. The

171

172

173

174

175

176

177

178

179

180

181

182

183

184

185

186

187

188

189

190

191

192

193

194

195

196

197

198

199

200

201

202

203

forward/backward ratio of Group 100 worms decreased when the worms were moving inside the MF (Fig. 6C, WSR test $p$-value $=0.010$ ) and so did their stay ratio (Fig. 6D, WSR test $p$-value $=$ 0.007). Regarding the effect of particles independently of MF, worms of Group 1 had increased stay ratio compared to control animals, even when the MF was turned off (Fig. 6D, Kruskal-Wallis test for all groups in OFF state $p$-value $=0.045$, WSR test comparing Group C OFF and Group 1 OFF $p$-value $=0.005)$. The speed of worms fed with $100 \mathrm{~nm}$ particles decreased when MF was on (Fig. 6I, WSR test $p$-value $=0.004$ ), as did speed $x$ for Group 100 (Fig. 6G, WSR test $p$-value $=0.016$ ) and for Group 1 worms (Fig. 6G, WSR test $p$-value $=0.037$ ), whereas speed y component did not change significantly for any group tested (Fig. 6H). Velocity was not affected in any of the groups tested (Fig. 6E and 6F).

We also examined two established path describing features, path curvature and range. The path curvature was not affected by either the presence of particles in the worm body or by MF under the experimental conditions applied (Fig. 6J). However, the range traveled is smaller when worms of Group 1 were moving inside MF compared to the range traveled when worms of the same group were moving without the effect of MF (Fig. 6K, WSR test $p$-value $=0.019$ ). This difference was not reflected in any of the distinct components of range ( $d_{x}$ and $d_{y}$, Figs. $6 \mathrm{~L}$ and $6 \mathrm{M}$, respectively).

The $p$-values for all comparisons are provided in Tables S1 and S2.

\section{Discussion}

\section{The impact of internalized nanoparticles on $C$. elegans locomotion}

Metal nanoparticles of various types have been used to evaluate particle toxicity using $C$. elegans (Gonzalez-Moragas et al., 2017; Gonzalez-Moragas et al., 2015b; Wu et al., 2012; Lim et al., 2012). Particle coating and size, worm developmental stage and duration of exposure have been shown to affect translocation of particles in various tissues of the worm's body (Pluskota et al., 2009; Gonzalez-Moragas et al., 2017; Wu et al., 2012). Particles used in the present study are larger (Gonzalez-Moragas et al., 2017; Gonzalez-Moragas et al., 2015b; Wu et al., 2012; Lim et al., 2012) and worms have been exposed to them for a shorter period (18-20hrs) than elsewhere (Wu et al., 2012; Yang et al., 2017). These differences may explain why most of the nanoparticles are found along the worms' pharynx, upper intestine (Fig. 4A, 4B) and lower intestine area (Fig. 4B). The location of $40 \mathrm{~nm}$ particles in worms of Group 40 around the pharynx and grinder area (Fig. 4C) is only approximate, as some deformation has been induced on the 
204

205

206

207

208

209

210

211

212

213

214

215

216

217

218

219

220

221

222

223

224

225

226

227

228

229

230

231

232

233

234

235

236

237

sample during preparation, and SEM allows for detecting objects that are close to the body surface. Particles aggregates are also found in the intestine (Fig. 5D), intestine lumen (Fig. 5E) and in muscle tissue (Fig. 5F).

Regarding locomotion features, the number of body bends in C. elegans L4 larvae has been shown to decrease significantly after exposure for $24 \mathrm{~h}$ to $9 \mathrm{~nm}$ nanoparticles coated with organic acid (Wu et al., 2012). Worms used in the present work are young adults and not larvae (Pluskota et al., 2009; Yang et al., 2017), which, as developing organisms, could be more vulnerable to toxic effects (Donkin and Williams, 1995). Moreover, particles used in the present work are larger (Gonzalez-Moragas et al., 2017; Gonzalez-Moragas et al., 2015b; Wu et al., 2012; Lim et al., 2012), and made of different metals (Lim et al., 2012; Li et al., 2012), which may result in different ability to overcome the intestine barrier or translocate to other tissues, as well as to different toxicity per se. Indeed, in our experiments, exposure of young adults to nanoparticles does not seem to massively affect posture, motion or path features (Fig. 6A-6M).

In our experiments, the only metrics that was affected by nanoparticles alone was the stay ratio for Group 1 (0.25, Fig. 6D), which was higher compared to Group C (0.12, Fig. 6D, comparison indicated by dashed line). This means that Group 1 worms remain paused for longer over the total time recorded, compared to Group C. Aggregates of $1 \mu \mathrm{m}$ particles in the intestine lumen (Fig. 4A) may resulted in heavier or more cumbersome worms, thus making it more difficult for them to move, although no difference to Group $C$ worms speed and velocity was detected (Fig. 6E-6I). Since there was no MF present, no magnetic effect on the locomotive machinery could take place (see also next section), leaving the locomotion speed unaffected. However, the mass of the particles themselves, or the friction generated between the now heavier worms and the agar surface, could be one possible cause of the observed increased pausing (a single adult C. elegans mass is $\sim 1 \mu \mathrm{g}$ (Muschiol et al., 2009), and according to Methods, a single worm may have ingested $1 \mu \mathrm{m}$ nanoparticles up to $10-15 \%$ of its body mass). Other effects on $C$. elegans physiology, e.g. impact on muscle function or increased body stiffness, could also be responsible for the increased pausing observed in Group 1 animals. Exploring these issues, however, lies beyond the scope of this paper.

\section{The impact of internalized nanoparticles, combined with MF, on C. elegans locomotion}

Magnetotaxis in C. elegans was recently demonstrated (Vidal-Gadea, 2015), with the participation of AFD neurons, the first to be identified as magnetosensory. It was suggested that endogenous magnetic material, previously reported in C. elegans, may be also involved (VidalGadea, 2015; Cranfield et al., 2004). These findings have sparked an ongoing discussion in the 
C. elegans community (Landler et al., 2018; Vidal-Gadea et al., 2018). In our experiments, the locomotive behavior of Group C worms, which did not contain any particles, was not affected by the externally applied MF (Fig. 6). However, the presence of $100 \mathrm{~nm}$ and, in some cases, $1 \mu \mathrm{m}-$ diameter internalized nanoparticles had an impact on specific locomotion features (Fig. 6), when MF was applied.

Group 100 worms that were moving in MF displayed more body bends (Fig. 6B) and spent more time paused (Fig. 6C, 6D). More body bends possibly indicate a more W-shaped locomotion, which has been described previously in burrowing worms, as opposed to the Sshaped crawling or C-shaped swimming motion (Beron et al., 2015). During burrowing, worms have to put effort to move inside a viscous medium. Hence, one could assume that Group 100 animals crawling in MF integrate more bends to their locomotion, to apply more effort to push their way forward. Indeed, counting the number of body bends has been suggested as a direct measure of the effort a worm is making to move (Hart, 2006). The assumption that moving may be laborious for these animals could be also supported by the fact that they pause more often (Fig. 6C, 6D) and they move more slowly (Fig. 6l). This is observed particularly in the direction of the MF ( $x$ direction, Fig. 2, Fig. 6G). The magnetic moment for the particles was aligned with the direction of the MF (on the plate surface they both follow the $x$ direction, Fig. 3F). (speed $_{x}$, Fig. 6G), which means that their locomotion was affected especially on the direction parallel to the MF. Moreover, Group 1 worms traveled over a smaller range (Fig. 6K), when the MF was on, which could reflect a modified exploratory behavior (Gray et al., 2005; Cheung et al., 2005). This is more likely to have happened due to changes in the locomotive status related to MF rather than to their incentive to explore, since other environmental factors (e.g., food abundance, temperature) did not change.

It is possible that worms slowed down when they found themselves in a particular orientation inside the MF, or when the internalized particles obtained a particular orientation with regard to the MF. This is supported by the results presented in Figure 2, where it is shown that the properties of MF change significantly in the direction of the MF. Therefore, any effect the MF may had on the particle-fed worm or on the internalized particles themselves, was changing as the worm was moving along the MF. due to the smaller MF or smaller gradient of MF of the particles in this group. However, we have been able to estimate the MF and the gradient of MF only for the $1 \mu \mathrm{m}$ particles, due to lack of available information on the magnetic properties of the $40 \mathrm{~nm}$ and the $100 \mathrm{~nm}$ particles. 
272 Nonetheless, we can compare the MF and gradient of MF between the particles based on their 273 size (see Supplementary information). We found that smaller particles have larger gradient of MF 274 compared to larger particles in their proximity. The overall impact each particle type has on the 275 worms' physiology depends on the magnitude and the gradient of MF. Both depend on the 276 material properties of the particles, which determine the magnetic moment. The experimental 277 observations suggested that the stronger effect among the three studied particles occurred in the 278 case of $100 \mathrm{~nm}$ particles.

The particles coating (Table 1) was not expected to affect their magnetic behavior. It could, however, impact their interaction with cells. Since the magnetic and physical properties of the particles are the most influential regarding the secondary MF effects, we focused on the particle size for our data analysis. In addition, the thickness of the coating was small (a single monolayer of streptavidin for the $1 \mu \mathrm{m}$ particles and a 2-3nm thick layer of polymer for the $40 \mathrm{~nm}$ particles, according to the manufacturers), which means that the magnetic core could still affect cells and tissues close to the particle. The experimental procedure did not allow us to know the quantity of particles ingested by each individual worm, nor the exact location and the precise interactions of particle aggregates in each individual. Therefore, we cannot extract conclusions based on the particles' quantity or exact aggregate location inside each worm tested.

Ideally, results shown in Figures 2 and 3 should be combined to assess the synergistic properties of the external component of the MF (generated by the electromagnets) and of the secondary component of the MF (generated by the particles in their vicinity). However, the MF generated by the particles is very localized (in the microscale), as shown in Figure 3. Thus, we discuss below the potential effects of the MF induced by the electromagnets and of the secondary MFs separately, and on their own respective scale. We also discuss the effect of particles

\section{Potential impact of internalized particles, combined with external MF, on C. elegans tissues}

The forces that were created either by the external MF or the paramagnetic particles themselves were small (Fig. 3E), and they were not strong enough to mechanically push the worms to move along their line of action. Hence, there must be some other mechanism responsible for the detected changes in worms' locomotion. The magnitude of the external and 302 secondary MFs had the same order of magnitude and they were small (Fig. 2 and 3A, 3C). 303 However, the gradient of the MF fields in the vicinity of the particles was substantially large (Fig. $3042 B, 2 D)$. 
Effects were likely to be more pronounced where the external MF was stronger, since that would result in stronger secondary MFs generated by the particles (until the magnetization of the particles becomes saturated). Hence, the locomotion of worms crawling under stronger MF, namely near the electromagnets (Fig. 2), was more likely to be affected, compared to worms that were moving where the external MF is weaker. Therefore, the spatial distribution of the MF can partly explain the variations observed in the experiments. Moreover, since we had no direct control over the location where particles resided in the worms' body, the presence or absence of paramagnetic particles where they could affect the mechanosensitive ion channels could be another reason for the variability that we observe in our experiments. In addition, the probability of a worm crawling into areas of higher magnetic flux, would result in it experiencing a stronger effect. The variations in the values of the metrics examined might in fact mirror that probability.

Zablotskii and colleagues (Zablotskii et al., 2016) provide several examples in which the gradient of MF can affect cellular and subcellular mechanisms. The gradient of the secondary MF fields obtained from our simulations (up to $2 \times 10^{5} \mathrm{~T} / \mathrm{m}$ for the $1 \mu \mathrm{m}$ particles, Fig. 3B, 3D) was well above the threshold that Zablotskii and colleagues (Zablotskii et al., 2016) suggest may impact cells with mechanosensitive ion channels $\left(10^{3} \mathrm{~T} / \mathrm{m}\right.$, see also Table 2 of Zablotskii et al., 2016). The gradient of the secondary internal MF fields was also above the threshold the same authors pose for magnetically induced changes on gene expression, however we consider such a possibility highly unlikely in our case, due to the very short time the external MF was applied ( $3.5 \mathrm{~min})$. Therefore, the secondary field generated by the paramagnetic particles upon application of external MF could lead to local gradients of MF inside the worm's body, large enough to interfere with the functionality of excitable cells (e.g., body muscle cells, see also Fig. 5F).

This could have happened by affecting the cells' ion channels, provided that the particles were very close or even in contact with the cells' membrane. Experimental data presented here cannot provide insight on exactly which cells might have been the target of the observed MF effect. However, TEM findings (Fig. 5) showed that particles aggregates could be located in body wall muscles (Fig. 5F). Therefore, the possibility that excitable, e.g. muscle cells, were affected by ingested particles in the presence of MF, is considerable. Moreover, impact on the intestine (Fig. 5D, 5E) could affect $C$. elegans physiology and locomotion dynamics, given the multiple roles of this complex tissue (Mcghee, 2007; Nagy et al., 2015). Further experiments are needed to clarify the mechanism behind the observed changes.

The possibility of MF having a direct action on the magnetosensitive neurons described in C. elegans by other authors (Vidal-Gadea, 2015) cannot be excluded. However, even if this were true, this action was not reflected in changed locomotion dynamics, which are the object of the 
present study. Note that in our experiments we did not have direct evidence of sensory or motor neurons being affected by MF, neither was that possibility explored.

Moreover, it is known that when magnetic field is applied to a population of paramagnetic particles, the particles self-organize into arrays, columns, or chains, depending on the nature of the applied magnetic field and the properties of the particle-containing medium (Liu et al., 2005; Liu et al., 1995; Doyle et al., 2002; Mirzakhalili et al., 2017). In our experiments, when the MF is turned $\mathrm{ON}$, it is likely that the internalized particles start moving, as they organize into selfassembled structures. Therefore, it is possible that this motion applies pressure on or stretches the surrounding tissue, resulting in disturbance of the normal locomotion pattern.

\section{Conclusions}

The effect of internalized paramagnetic nanoparticles, in combination with externally applied magnetic field, on the dynamics of $C$. elegans' locomotion is shown here. Established locomotion metrics, i.e. speed, motion state, bend count, showed differences between untreated worms and worms treated with particles when moving inside magnetic field, while they showed no difference between untreated and particles-treated worms in the absence of magnetic field. Possible explanations on the mechanism that leads to the observed results are provided by work on the effect of magnetic field gradients on cells (Zablotskii et al., 2016; Zablotskii et al., 2014), mediated by magnetic nanoparticles (Hughes et al., 2008), and by work on the self-organizing behavior of paramagnetic particles aggregates inside MF (Liu et al., 2005; Liu et al., 1995; Doyle et al., 2002). The exact mechanism by which the observed effect is achieved in the case of $C$. elegans needs to be further clarified. Our findings on the impact of internalized paramagnetic nanoparticles, in combination with externally applied magnetic field, on animals' behavior, could pave the way for more detailed studies on the sensitivity of biological systems to these biophysical factors. C. elegans nematodes could play a key role in the effort to decipher such phenomena.

\section{Materials and Methods}

\section{Nanoparticles internalization}

We investigated the locomotion of four groups of young adult wild type N2 C. elegans hermaphrodites, fed on (see also Table 1):
i) plain bacterial food source E. coli OP50, control animals-Group C, n=29; 
ii) E. coli OP50 mixed with $1 \mu$ m-diameter paramagnetic particles (Dynabeads MyOne Streptavidin C1, Invitrogen, Thermo Fisher Scientific, USA), Group 1, n=33;

iii) E. coli OP50 mixed with $100 \mathrm{~nm}$-diameter paramagnetic particles (nanomag-CLD-red, Micromod Partikeltechnologie $\mathrm{GmbH}$, Germany), Group 100, n=38 and

iv) E. coli OP50 mixed with $40 \mathrm{~nm}$-diameter paramagnetic particles (iron oxide nanocrystals, Ocean NanoTech, USA), Group 40, n=42. In all cases, particles were isolated from the initial suspension by brief centrifugation and were resuspended in OP50 in a final concentration of $0.5 \mathrm{mg} / \mathrm{ml}$ OP50-particle mix. Freshly made $60 \mathrm{~mm}$ standard NGM plates were seeded with $100 \mu \mathrm{l}$ of plain OP50 or OP50-particle mix. Plates were left to dry overnight in room temperature and $\sim 20$ worms were transferred in them the next day. Nematodes were left to feed on the plain or enriched bacterial lawn for $18-20 \mathrm{~h}$, at $20^{\circ} \mathrm{C}$. Then, they were either prepared for microscopy or 12-15 of them were transferred to a fresh, unseeded $35 \mathrm{~mm}$ NGM plate for locomotion recording. In the second case, worms were left to acclimatize in the new plate for $\sim 15$ min before recording. There were three reasons for transferring worms to a new, smaller plate. First, we wanted the worms to experience the effect of only internalized nanoparticles under MF and not of the remaining particles on the plate surface. Second, the presence of enriched bacterial lawn on the plate surface interfered with the tracking algorithm and could have affected the worms' locomotion due to its viscosity. Third, by using $35 \mathrm{~mm}$ plates we decreased the distance between the electromagnets and the worms (as shown in Fig. 1), so that the worms experience a stronger external MF.

For each group tested, experiments were run over 3 different experimental days. Therefore, each experimental day we processed 10-14 worms for a specific group. These 10-14 worms were treated simultaneously (on the same plate), and each one of them is considered a biological replicate.

\section{Fluorescent, Scanning and Transmission Electron Microscopy (SEM and TEM)}

Fluorescent Microscopy: Worms were transferred to an unseeded NGM plate and were washed with $0.5 \mathrm{ml}$ of $1 \mathrm{X}$ PBS. Next, they were transferred to a glass slide, where they were anesthetized on fresh agar pads (Shaham, 2006), using $10 \mathrm{mM} \mathrm{NaN}_{3}$ (Sulston, 1988). Samples

401 were imaged using a BX51WI Olympus fluorescent microscope (Olympus, Tokyo, Japan) coupled 402 with an ORCA-flash4.0 camera (Hamamatsu Photonics, Hamamatsu City, Japan).

Scanning Electron Microscopy: Samples were prepared as described previously (Hall et 404 al., 1999; Shaham, 2006), with modifications, dissection omitted. Briefly, worms were transferred 405 to an unseeded NGM plate and were washed with $0.5 \mathrm{ml}$ of $1 \mathrm{X}$ PBS. Next, they were transferred 
406

407

408

409

410

411

412

413

414

415

416

417

418

419

420

421

422

423

424

425

426

427

428

429

430

431

432

433

434

435

436

437

438

439

to a glass cover slip and were anesthetized using $10 \mathrm{mM} \mathrm{NaN}_{3}$ (Sulston, 1988). Samples were imaged using FEI Helios 650 nanolab SEM/FIB (FEI, Thermo Fisher Scientific, Waltham, MA).

Transmission Electron Microscopy: Samples were prepared based on the literature (Hall et al., 2012; Kovacs, 2015), with modifications. Briefly, tissues were fixed in pre-warmed or RT (room temperature) $3.2 \%$ PFA, $0.2 \%$ glutaraldehyde in $0.1 \mathrm{M}$ sodium cacodylate buffer for $1 \mathrm{~h}$ at RT and incubated overnight at $4{ }^{\circ} \mathrm{C}$. Next, they were rinsed $3 \times 10$ min with $0.1 \mathrm{M}$ sodium cacodylate buffer, and post-fix in $2 \%$ OsO4 in $0.1 \mathrm{M}$ sodium cacodylate buffer for $1 \mathrm{~h}$ at RT. Another rinse $3 \times 10 \mathrm{~min}$ with $0.1 \mathrm{M}$ sodium cacodylate buffer followed, and then worms were embedded in resin mold in histogel. Samples were dehydrated for 15 min each in 50\%, 70\%, $90 \%, 95 \%$, and finally two changes of $100 \%$ ethanol, cleared in two 15 min changes of propylene oxide, and infiltrated in propylene oxide:epon (Embed812), as follows: a.3:1, $1 \mathrm{~h}, \mathrm{~b} .1: 1,1 \mathrm{~h}, \mathrm{c}$. 1:3, $1 \mathrm{~h}, \mathrm{~d}$. Full strength, $2 \mathrm{~h}$ or overnight, two changes. Finally, samples were embedded in beam capsules in full epon, and polymerized at $60^{\circ} \mathrm{C}$ for $24 \mathrm{~h}$. Samples were imaged using a JEM-1400plus transmission electron microscope (JEOL, Peabody, MA).

\section{Worm Recording and Tracking}

Recording: A $35 \mathrm{~mm}$ plate containing worms of a specific group was placed between the two electromagnets, as shown in Figure 1, so that the plate surface and therefore the worms were positioned close to the center of the electromagnets. First, a $200 \mathrm{sec}$ movie (1 frame/sec) was recorded in the absence of MF (OFF state) and immediately after, a second $200 \mathrm{sec}$ movie (1 frame/sec) was recorded with the MF on (ON state), using QCapture Pro software, (QImaging, Surrey, Canada) and a Micropublisher3.3 RTV camera (QImaging, Surrey, Canada), mounted on an Olympus SZ61 microscope (Olympus, Tokyo, Japan). We recorded over 200 sec intervals because we were interested in detecting the transient effect of MF on locomotion dynamics. The two electromagnets used were a 4.0" Dia. Electromagnet, 12 VDC, and a 3.5" Dia. Electromagnet, 12 VDC, both from APW Company, Rockaway, NJ. Electromagnets were operated at 1.67 A and 3 A respectively, as indicated by the manufacturer, using a 1762 DC power supply (BK Precision, Yorba Linda, CA). By using a non-contact infrared thermometer (Omega Engineering, Norwalk, $\mathrm{CT}$ ) we verified that the plate surface temperature remained constant throughout the recording period.

Tracking: Every movie was imported to MATLAB (MathWorks, Natick, MA) for postprocessing. Each worm was tracked individually. To this end, we developed a custom tracking code in MATLAB (Fig. S2). In the first step, all frames were used to construct the movie background, which consisted of all the objects that did not move for long periods of time during 
440

441

442

443

444

445

446

447

448

449

450

451

452

453

454

455

456

457

458

459

460

461

462

463

464

465

466

467

468

469

470

471

472

the entire recording. Then, each frame was subtracted from the background to extract the foreground, which consisted of all moving objects. Next, the user was prompted with the initial frame of the movie, of which the background had been already subtracted, to select the worm to be tracked by the software. After the user selected the worm, the code created a small examining frame around it and excluded the targeted worm from the rest of the movie frame. Then, the cropped figure was converted to a binary image. After the binary image was enhanced, the shape of the binary object, i.e. the worm, and its global position were stored. Next, the code proceeded to the next movie frame and used the extracted global location of the worm as the center of the small examining frame. The small examining frame must be large enough to capture the motion of the worm in two successive movie frames. Since there was more than one worm freely moving in each experiment, there were occasions in which more than one object were included in the small examining frame. For such occasions, the user was prompted by the code to manually indicate again the worm to be tracked. This way the worm that was initially selected to be tracked was always encapsulated by the examining frame. The code continued the tracking process until the last frame of the movie was processed, and it stored the shape of the worm and its global location for each frame. Once finished, the user run the code again to track another worm.

\section{Locomotion Analysis}

The following features of $C$. elegans morphology and experimental setup properties were used for the quantification of $C$. elegans locomotion parameters.

\section{Morphology Features}

1. Length: The worm length was defined as the chain-code pixel length of worm skeleton, which was converted into $\mathrm{mm}$.

2. Centroid: The worm density was assumed to be constant throughout its body, so the centroid of mass was the same as geometric centroid. Since the swing of the head or tail (first or last $1 / 12$ chain-code length part of the worm) can significantly influence centroid determination, they were ignored when computing the centroid.

Setup

1. Coordinates system: The $x$ axis was set along the direction of the MF, between the two electromagnets, and $z$ axis was normal to the plate, pointing upwards (Fig.1). Thus, by applying the right-hand rule, the coordinates system was established. Since we did not identify head/tail orientation for the worms, the coordinates system was important for the detection of directionality. 
2. Unit Conversion: Any feature regarding length was derived first in pixels. With a known length recorded with the same experimental setup, the conversion between pixels and microns was determined.

Locomotion-related parameters of interest were divided in three categories: posture features, motion features and path features, as described extensively by Yemini and colleagues (Yemini et al., 2013), with minor modifications. A brief description of the examined features follows below.

\section{Posture Features}

1. Bends: The total body bends, measured in degrees, derive from the clockwise difference between two tangent supplementary angles (Fig. S4) along the worm skeleton. The mean value $\left(\right.$ mean $\left._{\text {bends }}\right)$ and standard deviation of bends $\left(s t d_{\text {bends }}\right)$ over the worm were also calculated, as intermediate steps.

2. Bend Count: This metric (bends $s_{\text {num }}$ ) corresponds to the number of bends along a single worm. First, the supplementary angles (see above, Bends) were computed along the worm skeleton. Next, a Gaussian filter over each 1/12 of the chain-code length of the skeleton was applied to the supplementary angles to smooth out any high frequency changes and is then normalized. The filter had a constant proportional to the reciprocal of the standard deviation, $\alpha=2.5$. By checking the sequence of supplementary angles, the bend count was incremented whenever the angle reaches $0^{\circ}$ or changes sign. The check started from the first $1 / 12$ segment to the last 1/12 segment to ignore small bends near the tail and the head.

\section{Motion Features}

1. Motion State: Worm's motion state can be divided in two types, the forward/backward state and the paused state. The worm was considered to be in the forward/backward state when its instantaneous speed was greater or equal to $5 \%$ of its mean length per second, and it was considered in the paused state when the instantaneous speed was less than $5 \%$ of its mean length per second. Therefore, the ratio of the time the worm was in the forward/backward state over the total recording time, namely the $f b_{\text {ratio }}$, and the ratio of the time the worm was in the paused state over the total recording time, namely the stay ratio stay $_{\text {ratio }}$, were calculated.

2. Velocity: Velocity is defined as the signed difference between a single worm's centroids of two sequential frames in the coordinate over the time gap between two frames (1sec). Velocity was further projected on two orthogonal axes $x$ and $y$ in the plane of the plate (Fig. 1), namely velocity $y_{x}$ and velocity. The absolute value of velocity and its components gave speed, speed $d_{x}$ and speed $_{y}$, respectively. 


\section{Path Features}

1. Path Curvature: This metric is defined as the angle, in radians, of the worm's path divided by the distance traveled, in microns. Three successive frames were used to approximate the start, middle and end of the worm's instantaneous path curvature. The angle was measured by the difference in tangent angles between the second to last frame centroid and the first to the second frame centroid. Then, the path curvature was obtained by dividing the angle by the distance between the first and last centroid.

2. Range: Range is defined as the distance between the worm's centroid and the centroid of the worm's path, in each frame. The range was projected onto the orthogonal axes $x$ and $y$ in the plane of the plate (Fig. 1) to obtain the x-range $d_{x}$, and the y-range $d_{y}$.

\section{Magnetic field characterization}

COMSOL Multiphysics (COMSOL, Burlington, MA) software was used to characterize the MF that is generated by the two electromagnets in the experimental setup. The data for the magnetic flux density of the electromagnets (available from the manufacturer) was used to calibrate the parameters of the electromagnets in COMSOL Multiphysics. The COMSOL Multiphysics model was used also to estimate the intensity of the external MF, the gradient of the external MF, and the forces that are applied on paramagnetic particles by the external MF. MATLAB was used to calculate the forces applied on the paramagnetic nanoparticles. More details are given in the Supplementary Information section.

\section{Statistical analysis}

Locomotion features were analyzed using non-parametric tests, since the AndersonDarling normality test $p$-value was $>0.05$ for all samples, thus rejecting the normality null hypothesis. For each metric analyzed, the Kruskal-Wallis test was used to detect whether there was any significant difference among the behaviors of all four groups in the absence of MF (OFF state). This comparison was done to determine whether the presence of particles themselves affects locomotion, regardless of external MF. Results were adjusted for ties and any difference was considered statistically significant when $p \leq 0.05$. To detect differences among worms of the same group, the Wilcoxon Signed Rank (WSR) test was used and differences were considered statistically significant when $p \leq 0.05$. All analyses were performed in Minitab (Minitab, State College, PA).

To design the experiment, we run a statistical power analysis, using $\mathrm{G}^{*}$ Power opensource software (Fig. S5). We prepared the sample size used in the experiments based on this 
541

542

543

544

545

546

547

548

549

550

551

552

553

554

555

556

557

558

559

560

561

562

563

564

565

566

567

568

569

570

571

572

573 estimation. In order to make sure that we would have enough worms, since some of them might be injured or lost during the process, we slightly increased the sample size number.

\section{Authors Contributions}

$B E$ and $E G$ conceived the idea; EG, EM and BE designed the experiments; $E G$ and $Y Z$ run experiments; $Y Z$ run tracking algorithm, processed and analyzed recordings; EM created tracking algorithm and run simulations; EG, YZ and EM collected and analyzed data; EG and EM wrote the paper, with input from YZ. All authors reviewed and edited the manuscript and gave final approval for publication.

\section{Acknowledgments}

We thank Nikos Chronis, Kenn Oldham and Jinhong Qu for the use of selected equipment, and Syeda Maisa for help with preliminary worm videos. Scanning electron microscopy was performed at the Electron Microbeam Analysis Laboratory (EMAL), with support from the University of Michigan College of Engineering; we thank John Mansfield, Kai Sun and Haiping Sun for the training. Transmission electron microscopy was performed at the Microscopy \& Image Analysis Laboratory (MIL) at the University of Michigan Medical School; we are grateful to Jeff Harrison and Pennelope Blakely for their help and guidance. We thank Surojit Sural for his valuable input on power analysis, and to Hannah Seidel and Elisa Frankel for feedback. We are particularly grateful to Hong Zhan for his generous help with TEM images interpretation.

\section{Competing interests: No competing interests declared.}

Funding: This work was supported by the National Sciences Foundation, Division of Civil, Mechanical and Manufacturing Innovation [award \#1334908 to B.E.], and the University of Michigan Office of Research [grant \#U055203 to E.G.]. 


\section{References}

575 Bae, J.-E., Bang, S., Min, S., Lee, S.-H., Kwon, S.-H., Lee, Y., Lee, Y.-H., Chung, J. \& Chae, K.-S. 2016. Positive geotactic behaviors induced by geomagnetic field in Drosophila. Molecular Brain, 9, 55.

Bansal, A., Zhu, L. J., Yen, K. \& Tissenbaum, H. A. 2015. Uncoupling lifespan and healthspan in Caenorhabditis elegans longevity mutants. Proceedings of the National Academy of Sciences, 112, E277-E286.

Belova, N. A. \& Acosta-Avalos, D. 2015. The Effect of Extremely Low Frequency Alternating Magnetic Field on the Behavior of Animals in the Presence of the Geomagnetic Field. Journal of Biophysics, 2015, 8.

Beron, C., Vidal-Gadea, A. G., Cohn, J., Parikh, A., Huong, G. \& Pierce-Shimomura, J. T. 2015. The burrowing behavior of the nematode Caenorhabditis elegans: A new assay for the study of neuromuscular disorders. Genes, brain, and behavior, 14, 357-368.

Cheung, B. H., Cohen, M., Rogers, C., Albayram, O. \& De Bono, M. 2005. Experience-dependent modulation of $C$. elegans behavior by ambient oxygen. Current Biology, 15, 905-917.

Cranfield, C. G., Dawe, A., Karloukovski, V., Dunin-Borkowski, R. E., De Pomerai, D. \& Dobson, J. 2004. Biogenic magnetite in the nematode caenorhabditis elegans. Proceedings in Biological Sciences, 271 Suppl 6, S436-S439.

De Bono, M. \& Bargmann, C. I. 1998. Natural Variation in a Neuropeptide Y Receptor Homolog Modifies Social Behavior and Food Response in C. elegans. Cell, 94, 679-689.

Donkin, S. G. \& Williams, P. L. 1995. Influence of developmental stage, salts and food presence on various end points using Caenorhabditis Elegans for aquatic toxicity testing. Environmental Toxicology and Chemistry, 14, 2139-2147.

Doyle, P. S., Bibette, J., Bancaud, A. \& Viovy, J.-L. 2002. Self-Assembled Magnetic Matrices for DNA Separation Chips. Science, 295, 2237-2237.

Fedele, G., Green, E. W., Rosato, E. \& Kyriacou, C. P. 2014. An electromagnetic field disrupts negative geotaxis in Drosophila via a CRY-dependent pathway. Nature Communications, 5, 4391.

Ghodbane, S., Lahbib, A., Sakly, M. \& Abdelmelek, H. 2013. Bioeffects of static magnetic fields: oxidative stress, genotoxic effects, and cancer studies. Biomedical Research International 2013, 602987. 
605 606

607

608

609

610

611

612

613

614

615

616

617

618

619

620

621

622

623

624

625

626

627

628

629

630

631

632

633

634

635

Gonzalez-Moragas, L., Roig, A. \& Laromaine, A. 2015a. C. elegans as a tool for in vivo nanoparticle assessment. Advances in Colloid and Interface Science, 219, 10-26.

Gonzalez-Moragas, L., Yu, S.-M., Benseny-Cases, N., Stürzenbaum, S., Roig, A. \& Laromaine, A. 2017. Toxicogenomics of iron oxide nanoparticles in the nematode C. elegans. Nanotoxicology, 11, 647657.

Gonzalez-Moragas, L., Yu, S.-M., Carenza, E., Laromaine, A. \& Roig, A. 2015b. Protective Effects of Bovine Serum Albumin on Superparamagnetic Iron Oxide Nanoparticles Evaluated in the Nematode Caenorhabditis elegans. ACS Biomaterials Science \& Engineering, 1, 1129-1138.

Gourgou, E., Chronis, N. 2016. Chemically induced oxidative stress affects ASH neuronal function and behavior in C. elegans. Scientific Reports, 6, 38147.

Gray, J. M., Hill, J. J. \& Bargmann, C. I. 2005. A circuit for navigation in Caenorhabditis elegans. Proceedings of the National Academy of Sciences of the United States of America, 102, 3184-3191.

Hahm, J. H., Kim, S., Diloreto, R. \& Shi, C. 2015. C. elegans maximum velocity correlates with healthspan and is maintained in worms with an insulin receptor mutation. Nature Communications, 6, 8919.

Hall, D. H., Hartwieg, E. \& Nguyen, K. C. 2012. Modern electron microscopy methods for C. elegans. Methods in Cell Biology, 107, 93-149.

Hall, D. H., Winfrey, V. P., Blaeuer, G., Hoffman, L. H., Furuta, T., Rose, K. L., Hobert, O. \& Greenstein, D. 1999. Ultrastructural Features of the Adult Hermaphrodite Gonad of Caenorhabditis elegans: Relations between the Germ Line and Soma. Developmental Biology, 212, 101-123.

Hart, A. C. 2006. Behavior. In: Ambros (ed.) Wormbook. The C. elegans Research Community.

Hedgecock, E. M. \& Russell, R. L. 1975. Normal and mutant thermotaxis in the nematode Caenorhabditis elegans. Proceedings of the National Academy of Sciences of the United States of America 72, 4061-4065.

Hong, F. T. 1995. Magnetic field effects on biomolecules, cells, and living organisms. Biosystems, 36, 187229.

Hsu, A.-L., Feng, Z., Hsieh, M.-Y. \& Xu, X. Z. S. 2009. Identification by machine vision of the rate of motor activity decline as a lifespan predictor in C. elegans. Neurobiology of aging, 30, 1498-1503.

Huang, H., Delikanli, S., Zeng, H., Ferkey, D. M. \& Pralle, A. 2010. Remote control of ion channels and neurons through magnetic-field heating of nanoparticles. Nature Nanotechnology 5, 602-606.

Hughes, S., Mcbain, S., Dobson, J. \& El Haj, A. J. 2008. Selective activation of mechanosensitive ion channels using magnetic particles. Journal of Royal Society Interface, 5, 855-863. 
636

637

638

639

640

641

642

643

644

645

646

647

648

649

650

651

652

653

654

655

656

657

658

659

660

661

662

663

664

665

Kale, P. G. \& Baum, J. W. 1980. Genetic effects of strong magnetic fields in drosophila melanogaster: II. Lack of interaction between homogeneous fields and fission neutron-plus-gamma radiation. Environmental Mutagenesis, 2, 179-186.

Khare, P., Sonane, M., Pandey, R., Ali, S., Gupta, K. C. \& Satish, A. 2011. Adverse Effects of TiO2 and ZnO Nanoparticles in Soil Nematode, Caenorhabditis elegans. Journal of Biomedical Nanotechnology, 7, 116-117.

Kim, J. H., Lee, S. H., Cha, Y. J., Hong, S. J., Chung, S. K., Park, T. H. \& Choi, S. S. 2017. C. elegans-on-a-chip for in situ and in vivo Ag nanoparticles' uptake and toxicity assay. Scientific Reports, 7, 40225.

Kovacs, A. L. 2015. The application of traditional transmission electron microscopy for autophagy research in Caenorhabditis elegans. Biophys Rep, 1, 99-105.

Kumari, K., Capstick, M., Cassara, A. M., Herrala, M., Koivisto, H., Naarala, J., Tanila, H., Viluksela, M. \& Juutilainen, J. 2017. Effects of intermediate frequency magnetic fields on male fertility indicators in mice. Environmental Research, 157, 64-70.

Landler, L., Nimpf, S., Hochstoeger, T., Nordmann, G. C., Papadaki-Anastasopoulou, A. \& Keays, D. A. 2018. Comment on "Magnetosensitive neurons mediate geomagnetic orientation in Caenorhabditis elegans". eLife, 7, e30187.

Lee, C.-H., Hung, Y.-C. \& Huang, G. S. 2010. Static magnetic field accelerates aging and development in nematode. Communicative \& Integrative Biology, 3, 528-529.

Lewczuk, B., Redlarski, G., Arkadiusz, Zikowska, N., Przybylska-Gornowicz, B., Krawczuk, M. 2014. Influence of Electric, Magnetic, and Electromagnetic Fields on the Circadian System: Current Stage of Knowledge. BioMed Research International, 2014, 13.

Li, G., Gong, J., Lei, H., Liu, J. \& Xu, X. Z. S. 2016. Promotion of behavior and neuronal function by reactive oxygen species in C. elegans. Nature Communications, 7, 13234.

Li, Y., Yu, S., Wu, Q., Tang, M., Pu, Y. \& Wang, D. 2012. Chronic Al2O3-nanoparticle exposure causes neurotoxic effects on locomotion behaviors by inducing severe ROS production and disruption of ROS defense mechanisms in nematode Caenorhabditis elegans. J Hazard Mater, 219-220, 221230.

Liedtke, W., Tobin, D. M., Bargmann, C. I. \& Friedman, J. M. 2003. Mammalian TRPV4 (VR-OAC) directs behavioral responses to osmotic and mechanical stimuli in Caenorhabditis elegans. Proceedings of the National Academy of Sciences, 100, 14531-14536. 
Lim, D., Roh, J. Y., Eom, H. J., Choi, J. Y., Hyun, J. \& Choi, J. 2012. Oxidative stress-related PMK-1 P38 MAPK activation as a mechanism for toxicity of silver nanoparticles to reproduction in the nematode Caenorhabditis elegans. Environ Toxicol Chem, 31, 585-592.

Liu, D., Maxey, M. R. \& Karniadakis, G. E. 2005. Simulations of dynamic self-assembly of paramagnetic microspheres in confined microgeometries. Journal of Micromechanics and Microengineering, 15, 2298.

Liu, J., Lawrence, E. M., Wu, A., Ivey, M. L., Flores, G. A., Javier, K., Bibette, J. \& Richard, J. 1995. FieldInduced Structures in Ferrofluid Emulsions. Phys Rev Lett, 74, 2828-2831.

Liu, J., Zhang, B., Lei, H., Feng, Z., Liu, J., Hsu, A. L., Xu, X. Z. 2013. Functional aging in the nervous system contributes to age-dependent motor activity decline in C. elegans. Cell Metab, 18, 392-402.

Long, X., Ye, J., Zhao, D. \& Zhang, S.-J. 2015. Magnetogenetics: remote non-invasive magnetic activation of neuronal activity with a magnetoreceptor. Science Bulletin, 60, 2107-2119.

Ma, H., Bertsch, P. M., Glenn, T. C., Kabengi, N. J. \& Williams, P. L. 2009. Toxicity of manufactured zinc oxide nanoparticles in the nematode Caenorhabditis elegans. Environ Toxicol Chem, 28, 13241330.

Malkemper, E. P., Eder, S. H. K., Begall, S., Phillips, J. B., Winklhofer, M., Hart, V. \& Burda, H. 2015. Magnetoreception in the wood mouse (Apodemus sylvaticus): influence of weak frequencymodulated radio frequency fields. Scientific Reports, 5, 9917.

Mcghee, J. D. 2007. The C. elegans intestine. In: Seydoux G. (ed.) WormBook. The C. elegans Research Community.

Meyer, J. N., Lord, C. A., Yang, X. Y., Turner, E. A., Badireddy, A. R., Marinakos, S. M., Chilkoti, A., Wiesner, M. R. \& Auffan, M. 2010. Intracellular uptake and associated toxicity of silver nanoparticles in Caenorhabditis elegans. Aquat Toxicol, 100, 140-150.

Mirzakhalili, E., Nam, W. \& Epureanu, B. I. 2017. Reduced-order models for the dynamics of superparamagnetic nanoparticles interacting with cargoes transported by kinesins. Nonlinear Dynamics, 90, 425-442.

Miyakoshi, J. 2005. Effects of static magnetic fields at the cellular level. Prog Biophys Mol Biol, 87, 213223.

Muschiol, D., Schroeder, F. \& Traunspurger, W. 2009. Life cycle and population growth rate of Caenorhabditis elegans studied by a new method. BMC Ecology, 9, 14-14.

Nagy, S., Huang, Y.-C., Alkema, M. J. \& Biron, D. 2015. Caenorhabditis elegans exhibit a coupling between the defecation motor program and directed locomotion. Scientific Reports, 5, 17174. 
Naito, M., Hirai, S., Mihara, M., Terayama, H., Hatayama, N., Hayashi, S., Matsushita, M. \& Itoh, M. 2012. Effect of a Magnetic Field on Drosophila under Supercooled Conditions. PLOS ONE, 7, e51902.

Nakata, K., Hu, Y., Uzun, O., Bakr, O. \& Stellacci, F. 2008. Chains of Superparamagnetic Nanoparticles. Advanced Materials, 20, 4294-4299.

Njus, Z., Feldmann, D., Brien, R., Kong, T., Kalwa, U., Pandey, S. 2015. Characterizing the Effect of Static Magnetic Fields on \&lt;i\&gt;C. elegans\&lt;/i\&gt; Using Microfluidics. Advances in Bioscience and Biotechnology, 06, 583-591.

Öcal, I., Kalkan, T., Ganay, O. 2008. Effects of alternating magnetic field on the metabolism of the healthy and diabetic organisms. Brazilian Archives of Biology and Technology, 51, 523-530.

Osipova, E. A., Pavlova, V. V., Nepomnyashchikh, V. A. \& Krylov, V. V. 2016. Influence of magnetic field on zebrafish activity and orientation in a plus maze. Behav Processes, 122, 80-86.

Parida, L., Neogi, S. \& Padmanabhan, V. 2014. Effect of Temperature Pre-Exposure on the Locomotion and Chemotaxis of C. elegans. PLOS ONE, 9, e111342.

Peliti, M., Chuang, J. S. \& Shaham, S. 2013. Directional Locomotion of C. elegans in the Absence of External Stimuli. PLOS ONE, 8, e78535.

Pierce-Shimomura, J. T., Chen, B. L., Mun, J. J., Ho, R., Sarkis, R. \& Mcintire, S. L. 2008. Genetic analysis of crawling and swimming locomotory patterns in C. elegans. Proceedings of the National Academy of Sciences of the United States of America, 105, 20982-20987.

Pluskota, A., Horzowski, E., Bossinger, O. \& Von Mikecz, A. 2009. In Caenorhabditis elegans NanoparticleBio-Interactions Become Transparent: Silica-Nanoparticles Induce Reproductive Senescence. PLOS ONE, 4, e6622.

Ramirez, E., Monteagudo, J. L., Garcia-Gracia, M. \& Delgado, J. M. R. 1983. Oviposition and development of Drosophila modified by magnetic fields. Bioelectromagnetics, 4, 315-326.

Shaham, S. W. 2006. Methods in Cell Biology, WormBook. In: Viktor Ambros (ed.) WormBook

Shaw, J., Boyd, A., House, M., Woodward, R., Mathes, F., Cowin, G., Saunders, M. \& Baer, B. 2015. Magnetic particle-mediated magnetoreception. Journal of the Royal Society Interface, 12, 0499.

Shcherbakov, D., Winklhofer, M., Petersen, N., Steidle, J., Hilbig, R. \& Blum, M. Magnetosensation in zebrafish. Current Biology, 15, R161-R162.

Shtonda, B. B. \& Avery, L. 2006. Dietary choice behavior in Caenorhabditis elegans. The Journal of experimental biology, 209, 89-102.

Sulston, J. E., Hodgkin, J. 1988. Methods. In: Wb (ed.) The Nematode Caenorhabditis elegans. Cold Spring Harbor, NY: Cold Spring Harbor Laboratory Press. 
730 731

732

733

734

735

736

737

738

739

740

741

742

743

744

745

746

747

748

749

750

751

752

753

754

755

756

757

758

759

Teodori, L., Grabarek, J., Smolewski, P., Ghibelli, L., Bergamaschi, A., De Nicola, M. \& Darzynkiewicz, Z. 2002. Exposure of cells to static magnetic field accelerates loss of integrity of plasma membrane during apoptosis. Cytometry, 49, 113-118.

Ueno, S., Lövsund, P. \& Öberg, P. Å. 1986. Effects of alternating magnetic fields and low-frequency electric currents on human skin blood flow. Medical and Biological Engineering and Computing, 24, 5761.

Vidal-Gadea, A., Bainbridge, C., Clites, B., Palacios, B. E., Bakhtiari, L., Gordon, V. \& Pierce-Shimomura, J. 2018. Response to comment on "Magnetosensitive neurons mediate geomagnetic orientation in Caenorhabditis elegans". elife, 7, e31414.

Vidal-Gadea, A., Ward, K., Beron, C., Ghorashian, N., Gokce, S., Russell, J., Truong, N., Parikh, A., Gadea, O., Ben-Yakar, A., Pierce-Shimomura, J. 2015. Magnetosensitive neurons mediate geomagnetic orientation in Caenorhabditis elegans. Elife, 4.

Wang, L., Du, H., Guo, X., Wang, X., Wang, M., Wang, Y., Wang, M., Chen, S., Wu, L. \& Xu, A. 2015. Developmental abnormality induced by strong static magnetic field in Caenorhabditis elegans. Bioelectromagnetics, 36, 178-189.

Wang, L., Wang, M., Du, H., Liu, Y. \& Xu, A. 2017. Lipid Metabolism was Interfered by PhosphatidylcholineCoated Magnetic Nanoparticles in C. elegans Exposed to 0.5 T Static Magnetic Field. Journal of Nanoscience and Nanotechnology, 17, 3172-3180.

Ward, A., Liu, J., Feng, Z. \& Shawn Xu, X. Z. 2008. Light-sensitive neurons and channels mediate phototaxis in C. elegans. Nature neuroscience, 11, 916-922.

Wu, Q., Li, Y., Tang, M. \& Wang, D. 2012. Evaluation of Environmental Safety Concentrations of DMSA Coated Fe(2)O(3)-NPs Using Different Assay Systems in Nematode Caenorhabditis elegans. PLoS ONE, 7, e43729.

Yang, Y.-F., Lin, Y.-J. \& Liao, C.-M. 2017. Toxicity-based toxicokinetic/toxicodynamic assessment of bioaccumulation and nanotoxicity of zerovalent iron nanoparticles in Caenorhabditis elegans. International Journal of Nanomedicine, 12, 4607-4621.

Yemini, E., Jucikas, T., Grundy, L. J., Brown, A. E. X. \& Schafer, W. R. 2013. A database of C. elegans behavioral phenotypes. Nature methods, 10, 877-879.

Zablotskii, V., Polyakova, T., Lunov, O. \& Dejneka, A. 2016. How a High-Gradient Magnetic Field Could Affect Cell Life. Scientific Reports, 6, 37407. 
Zablotskii, V., Syrovets, T., Schmidt, Z. W., Dejneka, A. \& Simmet, T. 2014. Modulation of monocytic leukemia cell function and survival by high gradient magnetic fields and mathematical modeling studies. Biomaterials, 35, 3164-3171.

\section{Figure and Table Legends}

Fig. 1: Experimental set up. A: Parts of the experimental set up for the application of gradient magnetic field on freely moving $C$. elegans. 1: Objective lens and camera; 2: Electromagnets; 3 : NGM plate with freely moving wild type N2 C. elegans, with schematic of plate orientation, red lines indicating $\mathrm{x}, \mathrm{y}$, and $\mathrm{z}$ axes; 4: Auxiliary transparent base; 5 : Working stage with bright light source; 6: Power Supply; 7: Computer and recording software. Objects are not depicted in scale. B: Schematic of the orientation of the NGM plate (pink circle), $x, y$, and $z$ axes and electromagnets (grey rectangles), top: view from above, bottom: view from aside.

\section{Fig. 2: COMSOL Multiphysics simulation results for the magnetic field generated by the} electromagnets. A: Overview of the magnetic field flux density on the plane of the worm plate surface. The arrows show the direction of the magnetic flux. B: The magnetic field flux density distribution on the worm plate surface. The arrows indicate the direction of the magnetic field (the component of the magnetic field in the perpendicular direction is set equal to zero to avoid arrows going in/out of the plane). C: The magnetic forces applied on particles located on the plane of the worm plate surface. The arrows show the direction of the magnetic forces (the component of the force in the perpendicular direction is set equal to zero to avoid arrows going in/out of the plane). $D$ : The gradient of the magnetic field in the direction of the axis that connects the centers of the two electromagnets.

\section{Fig. 3: Characterization of the magnetic field around the $1 \mu \mathrm{m}$ nanoparticles for two} different configurations. The direction of magnetic moment for both configurations is along the $x$ axis, as is shown in Figure 1. The magnetic moment of the particles is assumed to be similar and equal to the maximum value that is computed from the COMSOL Multiphysics simulations in the plate. A: The magnetic field flux density around three paramagnetic particles in the vertical configuration, i.e. along the y axis. The arrows indicate the direction of the magnetic field. B: The largest component of the gradient of the magnetic field for the vertical configuration of the paramagnetic particles. C: The magnetic field flux density norm around three paramagnetic 
793

794

795

796

797

798

799

800

801

802

803

804

805

806

807

808

809

810

811

812

813

814

815

816

817

818

819

820

821

822

823

824

825

826

particles in the horizontal configuration, i.e. along the $x$ axis. The arrows indicate the direction of the magnetic field. D: The largest component of the gradient of the magnetic field for the horizontal configuration of the paramagnetic particles. E: The forces between two particles in each configuration. F: The magnetic moment of the external magnetic field, which the particles experience once inside the magnetic field.

Table 1: Groups of worms tested and properties of the respective nanoparticles.

Fig. 4: Confirmation of nanoparticles uptake in young adult $C$. elegans. A: Internalization of $1 \mu \mathrm{m}$ paramagnetic particles is verified by bright field microscopy. Left: worm fed with plain $E$. coli OP50 (control, Group C). Right: worm fed with E. coli OP50 mixed with 1um particles. Particles appear to be aggregated in the dark-colored pharynx (PHX) and intestine (INT) of Group 1 worms, in contrast to the light-colored pharynx (PHX) and intestine (INT) of Group C worms. Scale bar: $0.1 \mathrm{~mm}$. B: Internalization of $100 \mathrm{~nm}$ magnetic, fluorescent nanoparticles is verified by epifluorescent microscopy. Top panels: worm fed with plain E. coli OP50 (control, Group C), bottom panels: worm fed with E. coli OP50 mixed with $100 \mathrm{~nm}$ particles. Bright light: worms illuminated by bright light source; rhodamine: worms visualized with optical filter for rhodamine, Excitation $545 \mathrm{~nm} /$ Emission $565 \mathrm{~nm}$; GFP: worms visualized with optical filter for green fluorescent protein (GFP), Excitation $395 \mathrm{~nm} /$ Emission $510 \mathrm{~nm}$; DAPI: worms visualized with optical filter for DAPI, Excitation $358 \mathrm{~nm} /$ Emission $460 \mathrm{~nm}$. In GFP and DAPI images, autofluorescence is the only fluorescence detected. Scale bar: $0.1 \mathrm{~mm}$. C: Internalization of 40 $\mathrm{nm}$ paramagnetic particles is verified by scanning electron microscopy (SEM). Left: a whole $C$. elegans as captured by SEM, using Everhart-Thornley SE detector. Center: $40 \mathrm{~nm}$ particles, shown as white dots, detected close to $C$. elegans pharynx, using circular backscatter (CBS) detector, magnification 1500x. Right: $40 \mathrm{~nm}$ particles, shown as white dots, detected close to $C$. elegans pharynx, using circular backscatter (CBS) detector, magnification 3000x. Location of particles is approximate, due to distortion generated during sample processing.

\section{Fig. 5: Location of nanoparticles in young adult C. elegans, using Transmission Electron} Microscopy (TEM). A-B-C: Free particles (A: $100 \mathrm{~nm}, \mathrm{~B}: 1 \mu \mathrm{m}, \mathrm{C}: 40 \mathrm{~nm}$ ) imaged with TEM; D: $40 \mathrm{~nm}$ particles aggregates in the intestine; $\mathrm{E}: 100 \mathrm{~nm}$ particles aggregates in the intestine lumen; $\mathrm{F}: 40 \mathrm{~nm}$ particles aggregate in body muscle tissue (m.t.), close to epidermis (e) and cuticle (c). Dotted circles indicate particles aggregates in all panels. 
Fig. 6: Locomotion features and their metrics, as they have been quantified for all four groups of worms tested (Group C: control animals, fed on plain food source $E$. coli OP50, n=29; Group 1: fed on E. coli OP50 mixed with $1 \mu$ m-diameter paramagnetic particles, n=33; Group 100: fed on E. coli OP50 mixed with $100 \mathrm{~nm}$-diameter iron core paramagnetic particles, $\mathrm{n}=38$; and Group 40: fed on E. coli OP50 mixed with $40 \mathrm{~nm}$-diameter iron core paramagnetic particles, $\mathrm{n}=42$ ) in the absence (OFF state) or in the presence (ON state) of external magnetic field. A-B: Posture features, C-I: Motion features, J-M: Path features. For each group tested worms were tracked in 3 different experimental days. Grey dots represent individual worms; red diamonds represent the mean; blue boxes indicate the median confidence interval box, with a middle line indicating the median. Dashed lines show comparisons between worms of different groups in the absence of magnetic field (OFF state). Continuous lines show comparisons between the ON and OFF state of worms of the same group. All $p$-values given are calculated by the Wilcoxon Signed Rank test with confidence interval set at $95 \%$, and any difference was considered statistically significant when $p \leq 0.05$. The $p$-values for all comparisons are given in Tables S1 and S2.

Fig. S1: Calibration of the parameters in COMSOL Multiphysics simulations to match the available data for magnet $1(A)$ and magnet $2(B)$ that are used in the experiments.

Fig. S2: An overview of the steps that are taken to analyze the locomotion of the worms. Top to bottom: A worm is selected in the first frame to be tracked. Next, several image enhancements are performed on the subframe that is created around the selected worm. Next, the grayscale image is converted to a binary image and postprocessing, e.g. finding the centroid (blue circle) of the worm, is performed. All these steps are conducted for the whole movie that is recorded. Finally, the data collected from all experiments are compared.

Fig. S3: All the components of the gradient of the magnetic field for the particles in the vertical (along the $y$ axis) and horizontal (along the $x$ axis) configuration.

Fig. S4: The supplementary angle $(\alpha)$ is the difference in tangent angles at each skeleton point.

Table S1: The magnetic field effect on the locomotion of worms with internalized nanoparticles. $p$-values of Wilcoxon Signed Rank test for all comparisons between OFF and ON state of each group studied. With bold are highlighted the statistically significant differences, with $p$-value $\leq 0.05$. 
862 Table S2: The particle effect on the locomotion of worms with internalized nanoparticles. In the

863 second column are given the $p$-values of Kruskal-Wallis test used to compare all groups of worms

864 studied during their OFF state. In the last three columns are given the $p$-values of the Wilcoxon

865 Signed Rank test used to compare Group C with each of the other three particle-containing

866 groups. The Wilcoxon Signed Rank test was run only when Kruskal-Wallis test gave a $p$-value

$867 \leq 0.05$. With bold are highlighted the statistically significant differences, with $p$-value $\leq 0.05$.

868

869 Fig. S5: Power analysis for locomotion dynamics experiments. G*Power open source software

870 was used as described in Methods. A. Plot that shows the probability of detecting a real effect

871 with regard to sample size; B. Table showing the protocol followed for the power analysis. The

872 mean of group1 was set to 0.5 and the mean of group2 was set to 0.4 , with SD within each group

$873 \sigma=0.1$. 


\section{Figure 1}

A

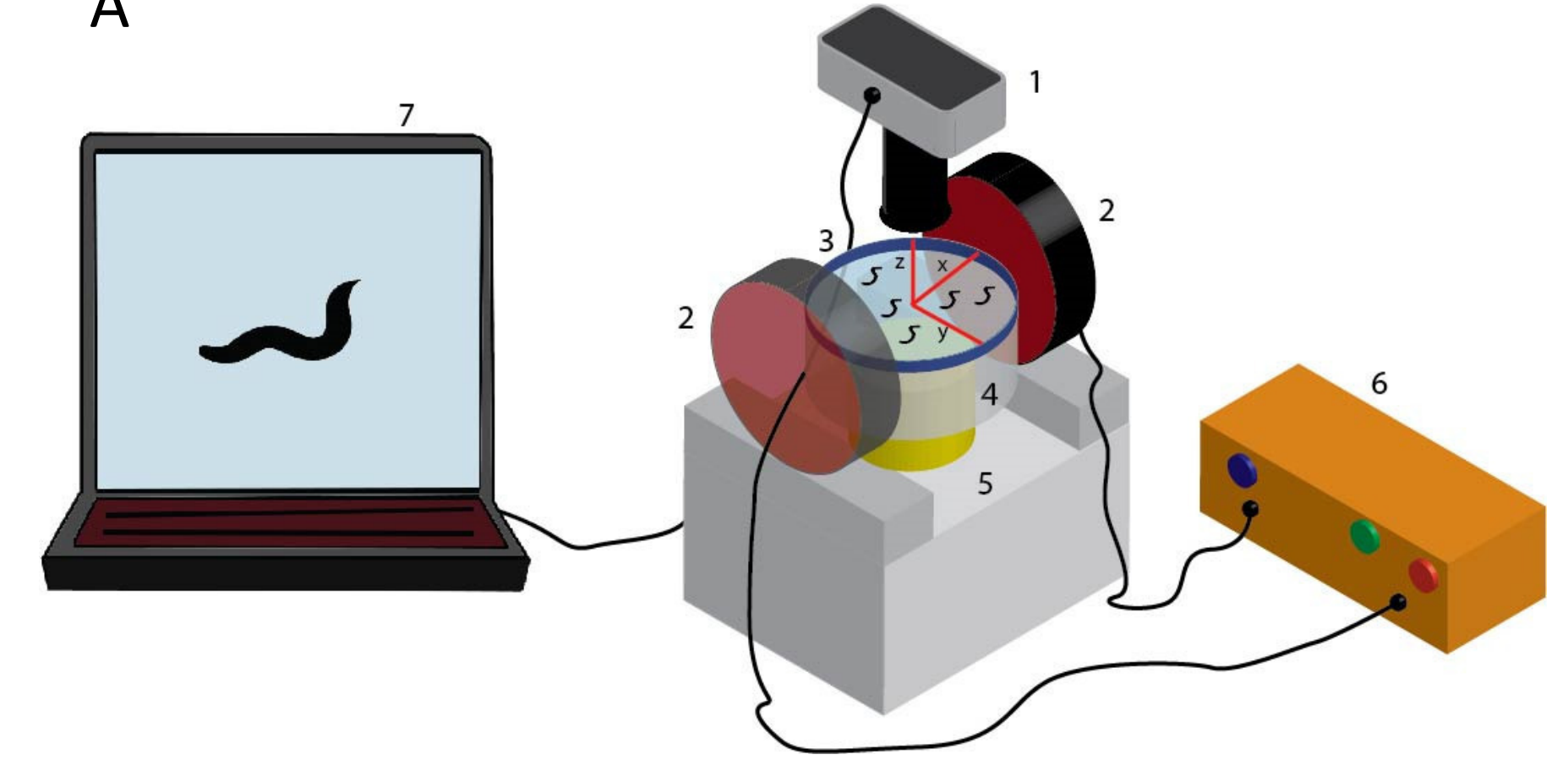

B
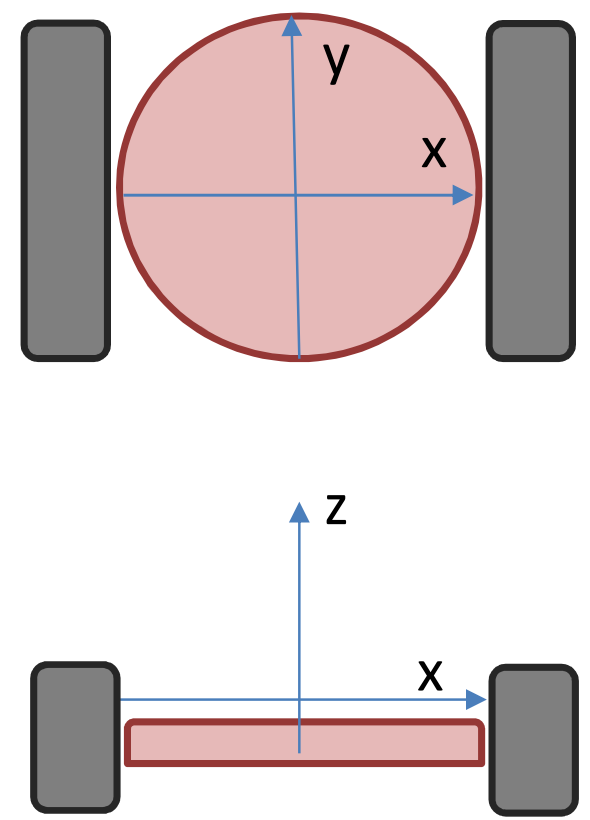

Fig. 1: Experimental set up. A: Parts of the experimental set up for the application of gradient magnetic field on freely moving $C$. elegans. 1: Objective lens and camera; 2 : Electromagnets; 3: NGM plate with freely moving wild type N2 C. elegans, with schematic of plate orientation, red lines indicating $x, y$, and $z$ axes; 4: Auxiliary transparent base; 5: Working stage with bright light source; 6: Power Supply; 7: Computer and recording software. Objects are not depicted in scale. B: Schematic of the orientation of the NGM plate (pink circle), x, y, and $z$ axes and electromagnets (grey rectangles), top: view from above, bottom: view from aside. 
Figure 2 Magnetic flux density norm [G]

A
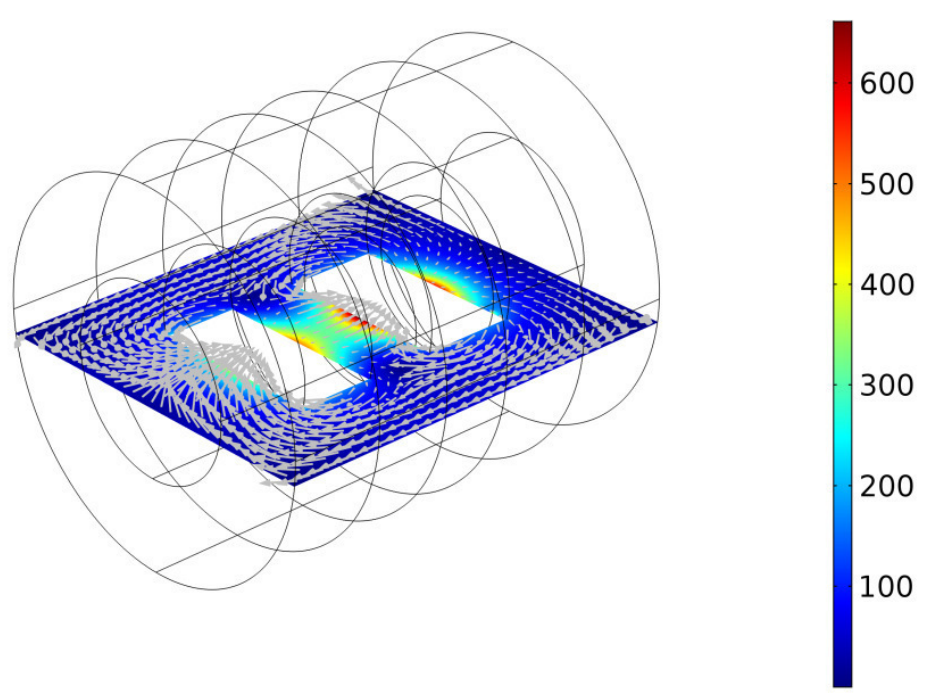

C

Force $[\mathrm{pN}]$

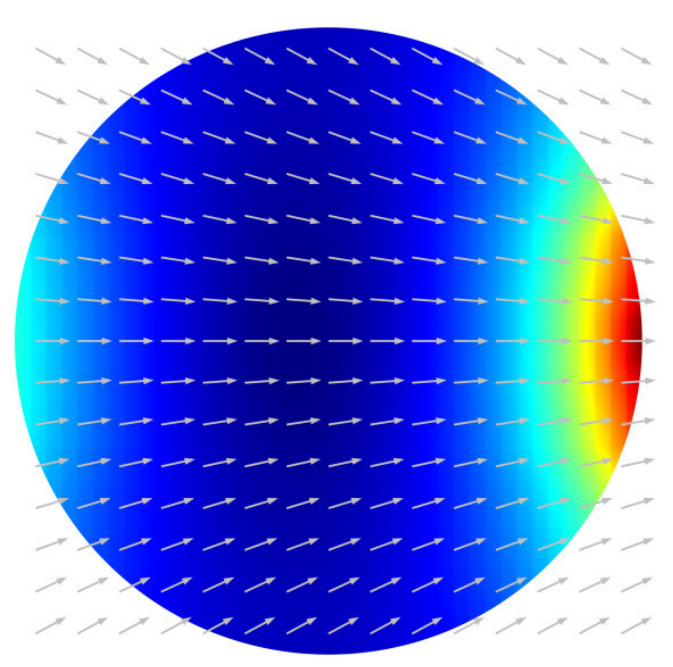

Magnetic flux density norm [G]

B

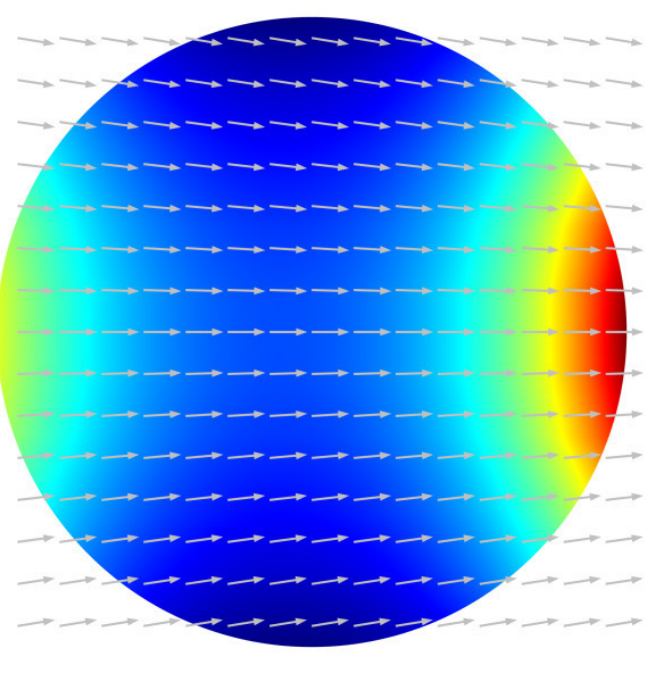

$\mathrm{Bxx}[\mathrm{T} / \mathrm{m}]$

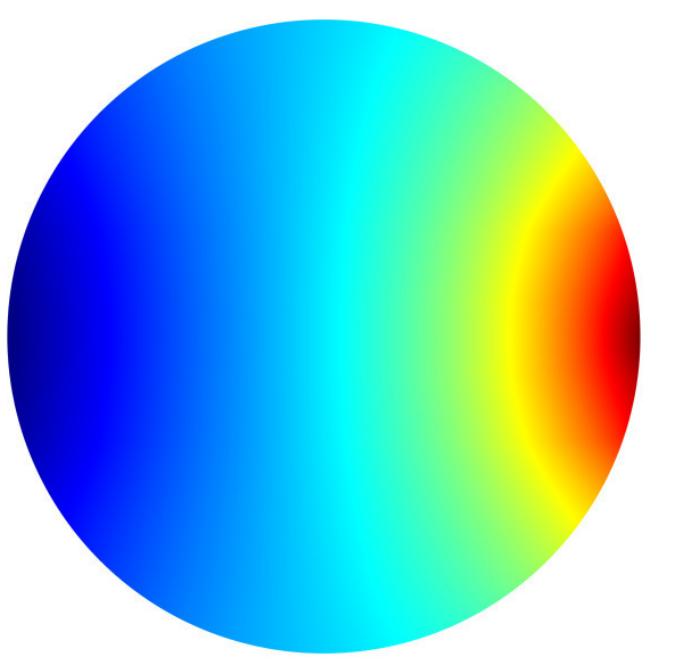

a

650
600
550
500
450
400
350
300
250

a

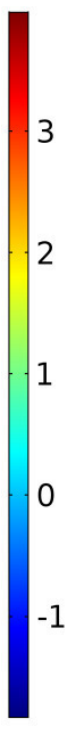


Fig. 2: COMSOL Multiphysics simulation results for the magnetic field generated by the electromagnets. A: Overview of the magnetic field flux density on the plane of the worm plate surface. The arrows show the direction of the magnetic flux. B: The magnetic field flux density distribution on the worm plate surface. The arrows indicate the direction of the magnetic field (the component of the magnetic field in the perpendicular direction is set equal to zero to avoid arrows going in/out of the plane). C: The magnetic forces applied on particles located on the plane of the worm plate surface. The arrows show the direction of the magnetic forces (the component of the force in the perpendicular direction is set equal to zero to avoid arrows going in/out of the plane). D: The gradient of the magnetic field in the direction of the axis that connects the centers of the two electromagnets. 


\section{Figure 3}

A

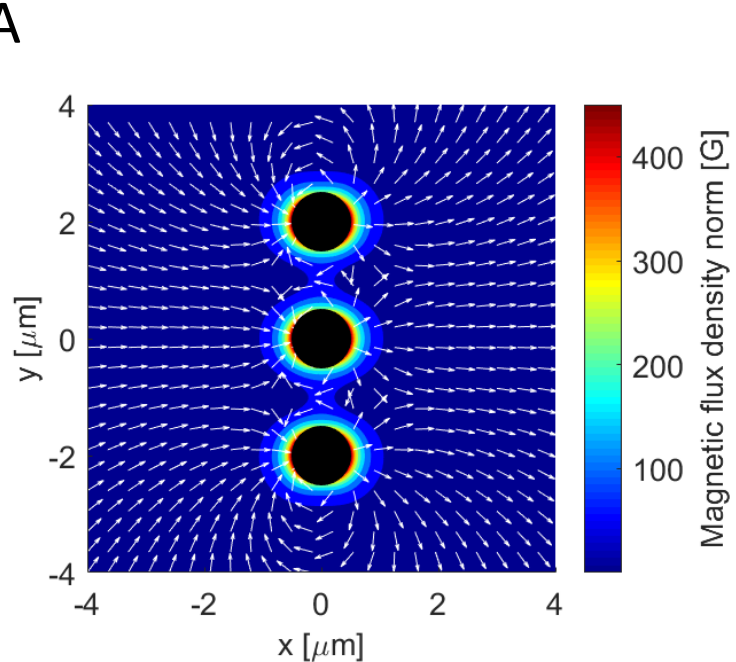

C

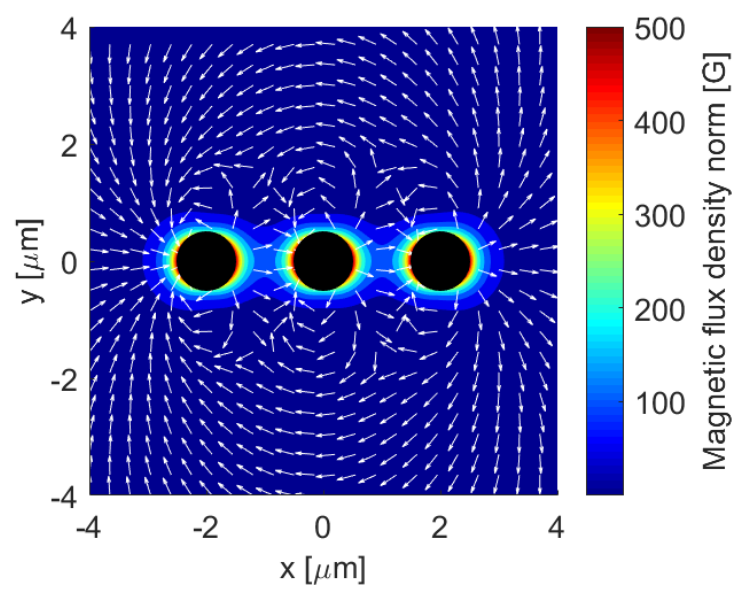

B

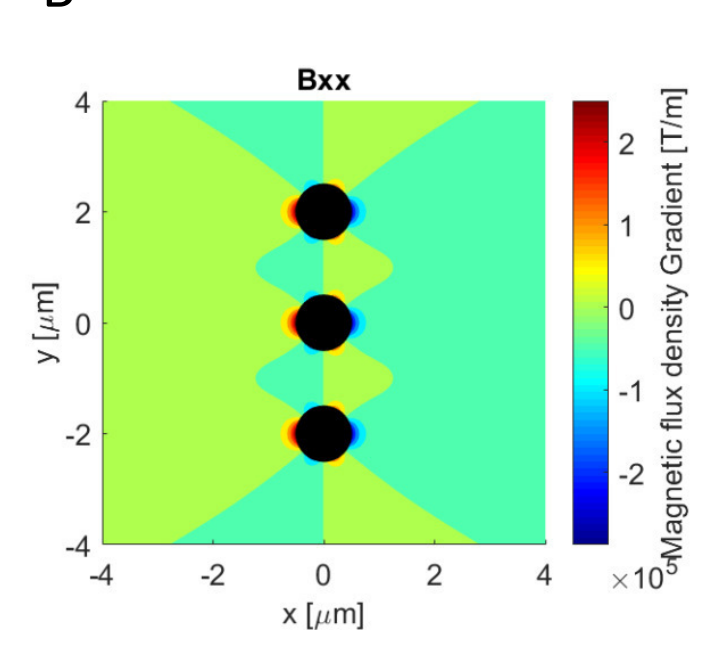

D

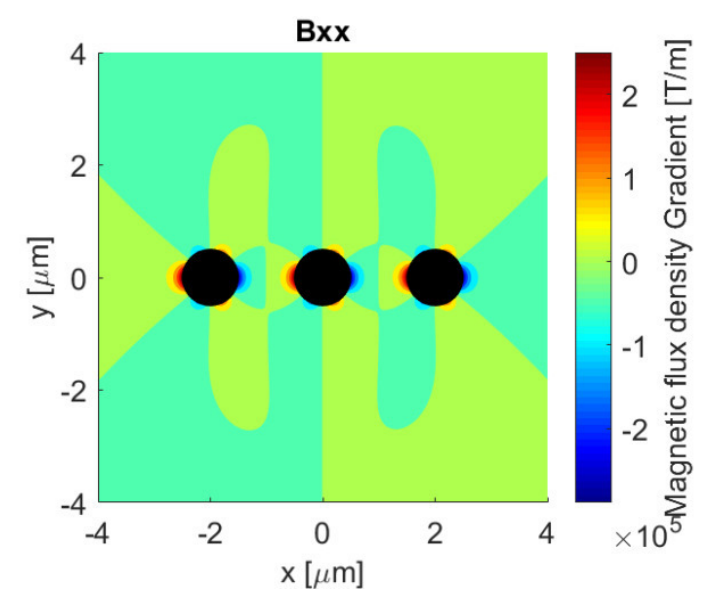

E

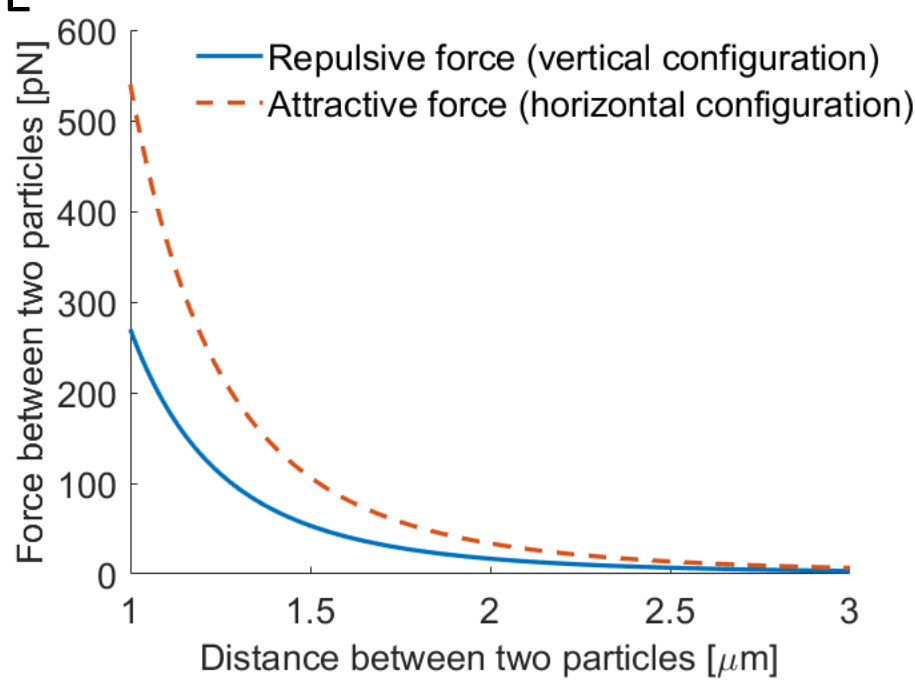

F

Magnetic moment $\left(m^{2} . A\right)$

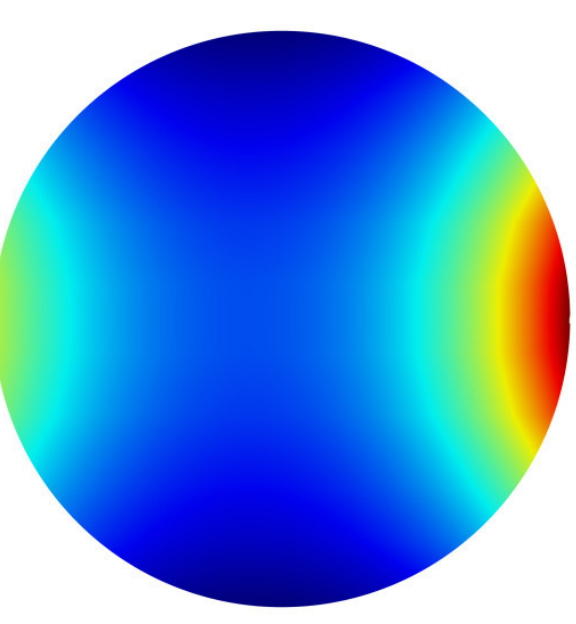

$\times 10^{-14}$ 
Fig. 3: Characterization of the magnetic field around the $1 \mu \mathrm{m}$ nanoparticles for two different configurations. The direction of magnetic moment for both configurations is along the $x$ axis, as is shown in Figure 1 . The magnetic moment of the particles is assumed to be similar and equal to the maximum value that is computed from the COMSOL Multiphysics simulations in the plate. A: The magnetic field flux density around three paramagnetic particles in the vertical configuration, i.e. along the $y$ axis. The arrows indicate the direction of the magnetic field. B: The largest component of the gradient of the magnetic field for the vertical configuration of the paramagnetic particles. C: The magnetic field flux density norm around three paramagnetic particles in the horizontal configuration, i.e. along the $x$ axis. The arrows indicate the direction of the magnetic field. D: The largest component of the gradient of the magnetic field for the horizontal configuration of the paramagnetic particles. E: The forces between two particles in each configuration. F: The magnetic moment of the external magnetic field, which the particles experience once inside the magnetic field. 


\begin{tabular}{ccccc}
\hline Group & Particle Size & Coating & Magnetic properties & Fluorescence \\
\hline Group C & - & - & - & - \\
Group 1 & $1 \mu \mathrm{m}$ & Streptavidin & Paramagnetic & No \\
Group 100 & $100 \mathrm{~nm}$ & No & Paramagnetic & Rhodamine \\
Group 40 & $40 \mathrm{~nm}$ & $-\mathrm{COOH}$ & Paramagnetic & No \\
\hline
\end{tabular}

Table 1: Groups of worms tested and properties of the respective nanoparticles. 
Figure 4

A

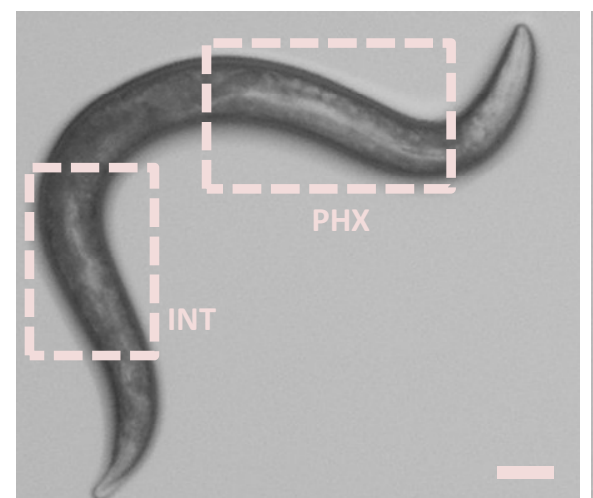

Group C

B

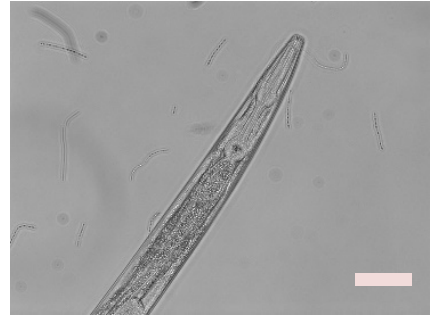

Bright light

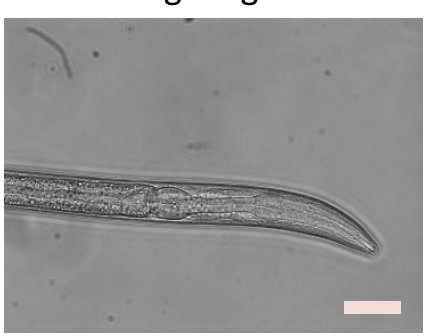

C

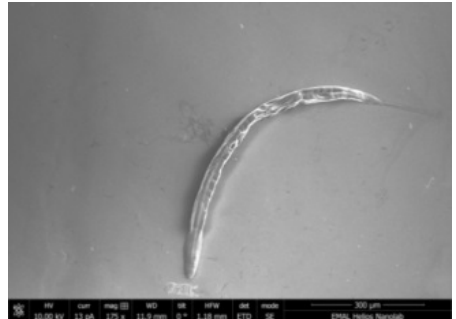

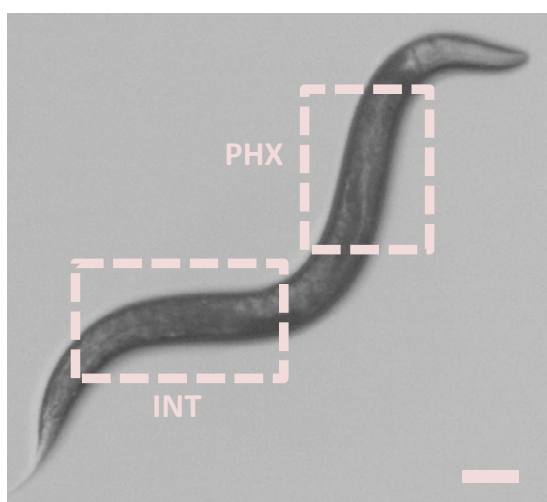

Group 1
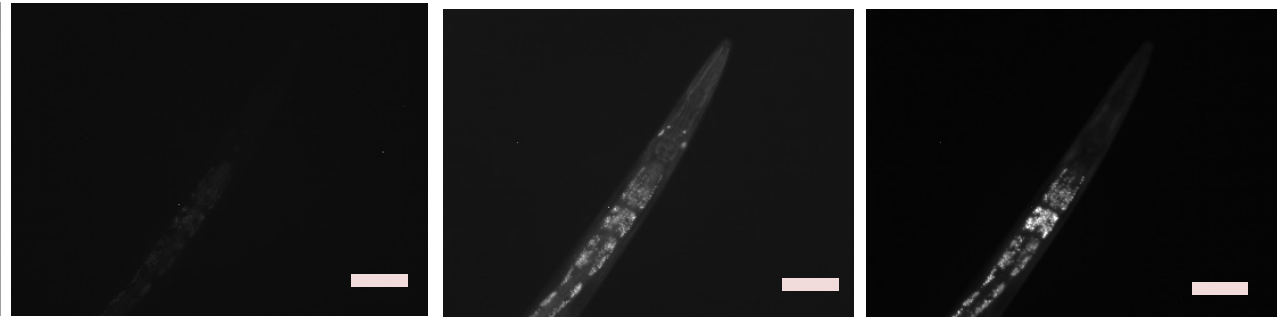

Group C

Rhodamine

GFP
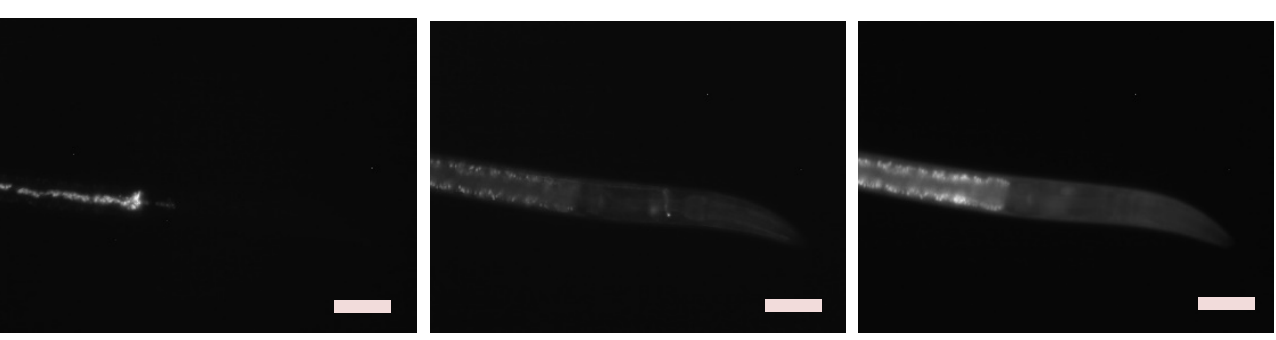

Group 100

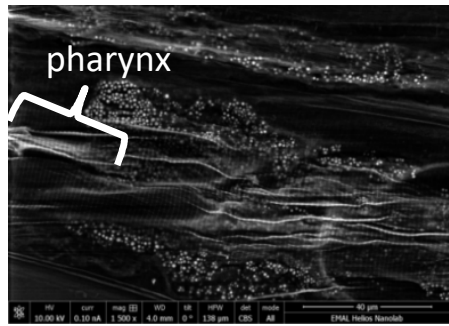

Group 40

Group 40 
Fig. 4: Confirmation of nanoparticles uptake in young adult $C$. elegans. A: Internalization of $1 \mu \mathrm{m}$ paramagnetic particles is verified by bright field microscopy. Left: worm fed with plain E. coli OP50 (control, Group C). Right: worm fed with E. coli OP50 mixed with 1um particles. Particles appear to be aggregated in the dark-colored pharynx (PHX) and intestine (INT) of Group 1 worms, in contrast to the light-colored pharynx (PHX) and intestine (INT) of Group C worms. Scale bar: 0.1mm. B: Internalization of $100 \mathrm{~nm}$ magnetic, fluorescent nanoparticles is verified by epifluorescent microscopy. Top panels: worm fed with plain E. coli OP50 (control, Group C), bottom panels: worm fed with E. coli OP50 mixed with $100 \mathrm{~nm}$ particles. Bright light: worms illuminated by bright light source; rhodamine: worms visualized with optical filter for rhodamine, Excitation $545 \mathrm{~nm} /$ Emission $565 \mathrm{~nm}$; GFP: worms visualized with optical filter for green fluorescent protein (GFP), Excitation $395 \mathrm{~nm} /$ Emission $510 \mathrm{~nm}$; DAPI: worms visualized with optical filter for DAPI, Excitation 358

$\mathrm{nm} /$ Emission $460 \mathrm{~nm}$. In GFP and DAPI images, autofluorescence is the only fluorescence detected. Scale bar: $0.1 \mathrm{~mm}$. C: Internalization of $40 \mathrm{~nm}$ paramagnetic particles is verified by scanning electron microscopy (SEM). Left: a whole $C$. elegans as captured by SEM, using Everhart-Thornley SE detector. Center: $40 \mathrm{~nm}$ particles, shown as white dots, detected close to C. elegans pharynx, using circular backscatter (CBS) detector, magnification 1500x. Right: $40 \mathrm{~nm}$ particles, shown as white dots, detected close to $C$. elegans pharynx, using circular backscatter (CBS) detector, magnification 3000x. Location of particles is approximate, due to distortion generated during sample processing. 


\section{Figure 5}
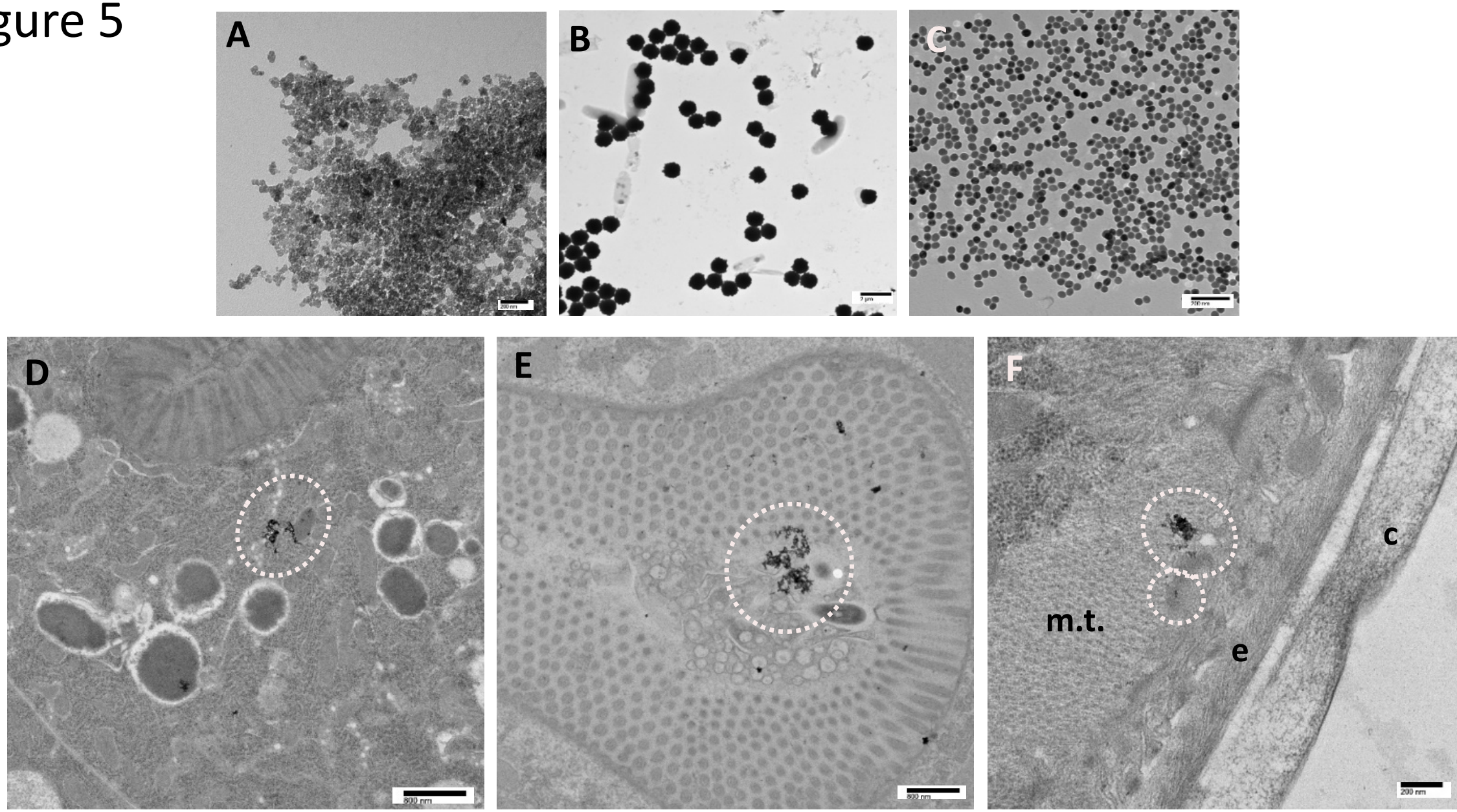

Fig. 5: Location of nanoparticles in young adult C. elegans, using Transmission Electron Microscopy (TEM). A-BC: Free particles (A: $100 \mathrm{~nm}, \mathrm{~B}: 1 \mu \mathrm{m}, \mathrm{C}: 40 \mathrm{~nm}$ ) imaged with TEM; D: $40 \mathrm{~nm}$ particles aggregates in the intestine; $\mathrm{E}: 100 \mathrm{~nm}$ particles aggregates in the intestine lumen; $\mathrm{F}: 40 \mathrm{~nm}$ particles aggregate in body muscle tissue (m.t.), close to epidermis (e) and cuticle (c). Dotted circles indicate particles aggregates in all panels. 
Figure 6

Posture Features (A-B)
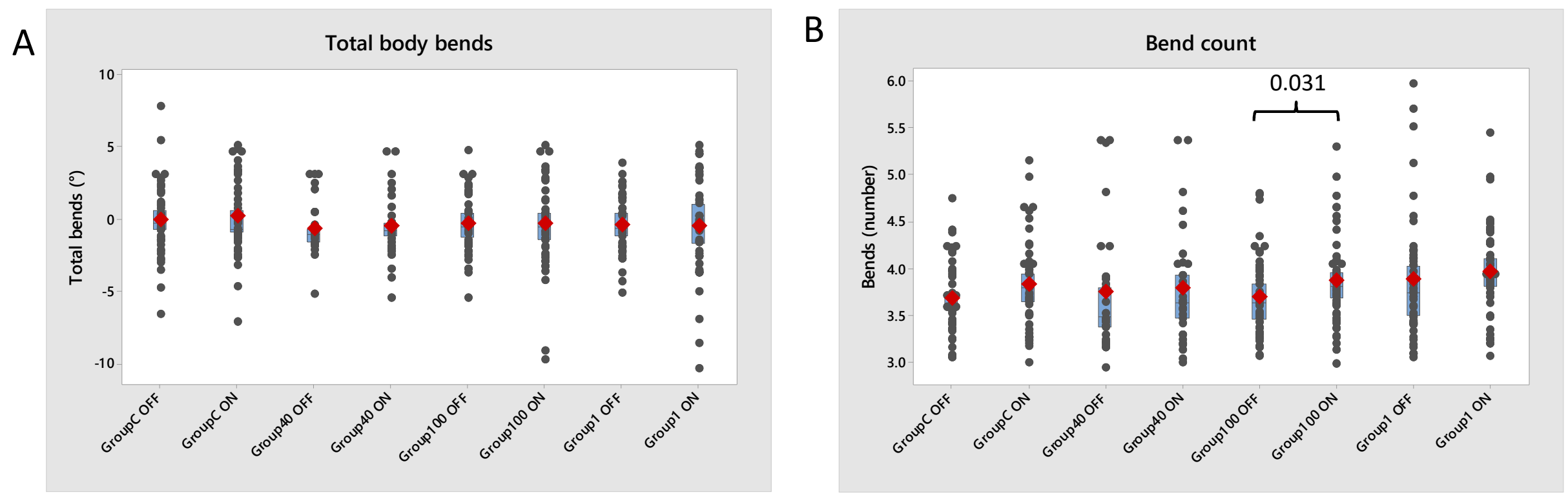
Figure 6 (continued)

\section{Motion Features (C-I)}

C

Fonward/backward state

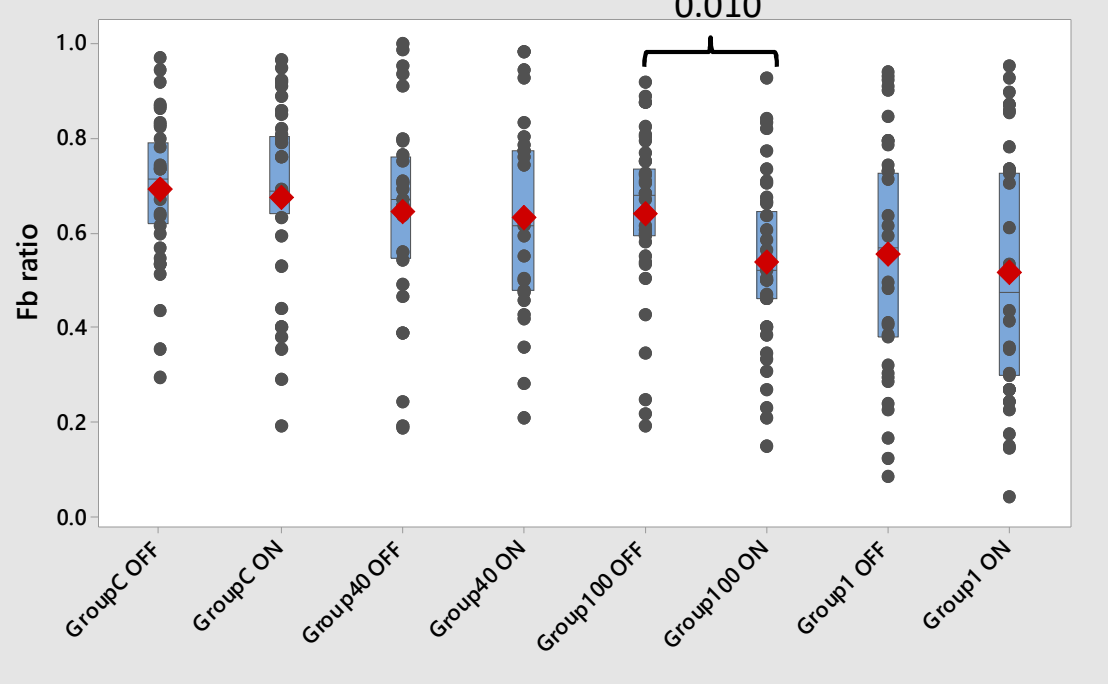

E

Velocity $x$ ( $x$ axis component of velocity)

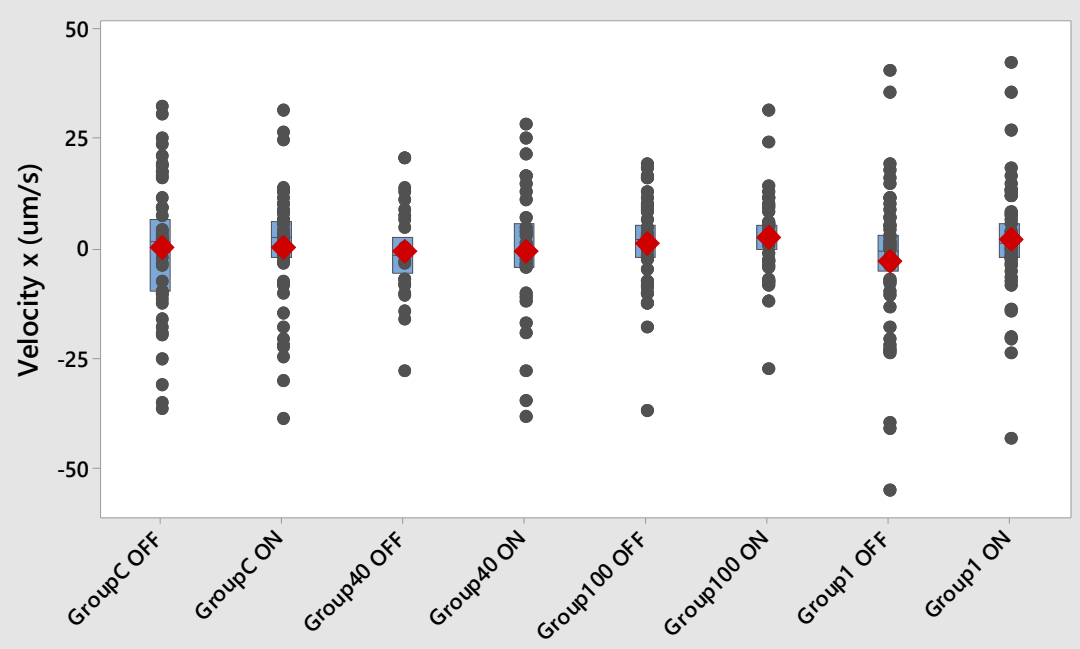

D

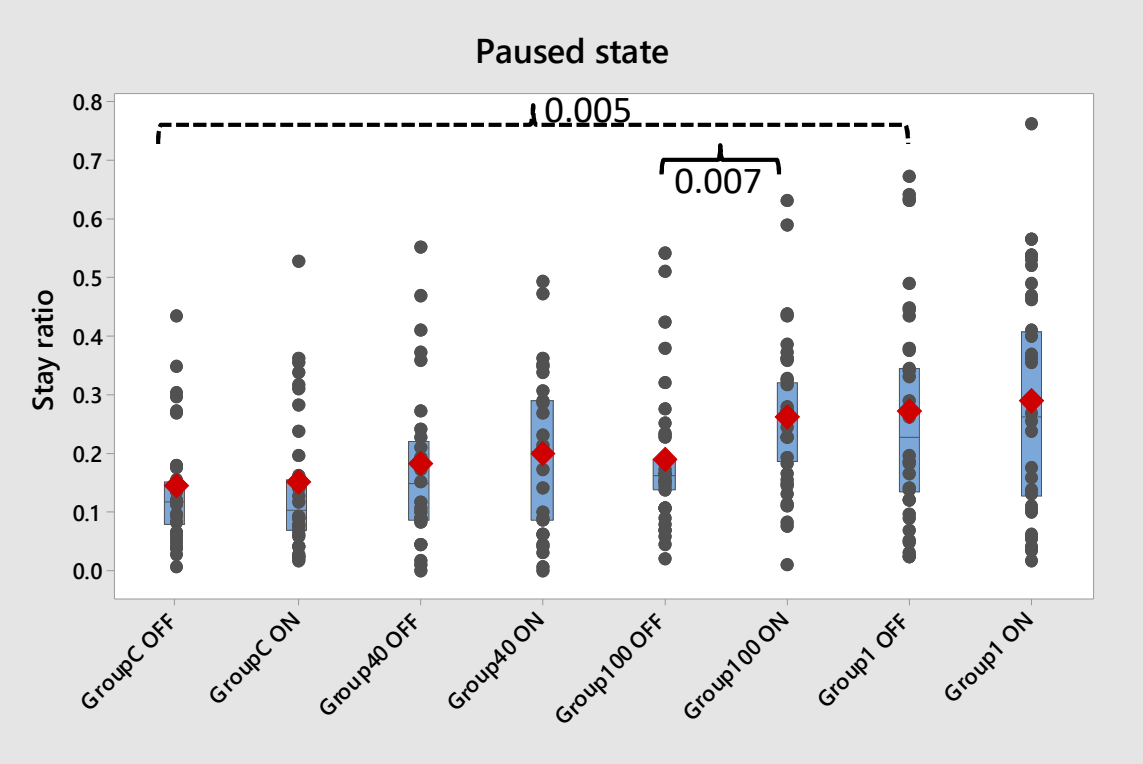

$\mathrm{F}$

Velocity y ( $y$ axis component of velocity)

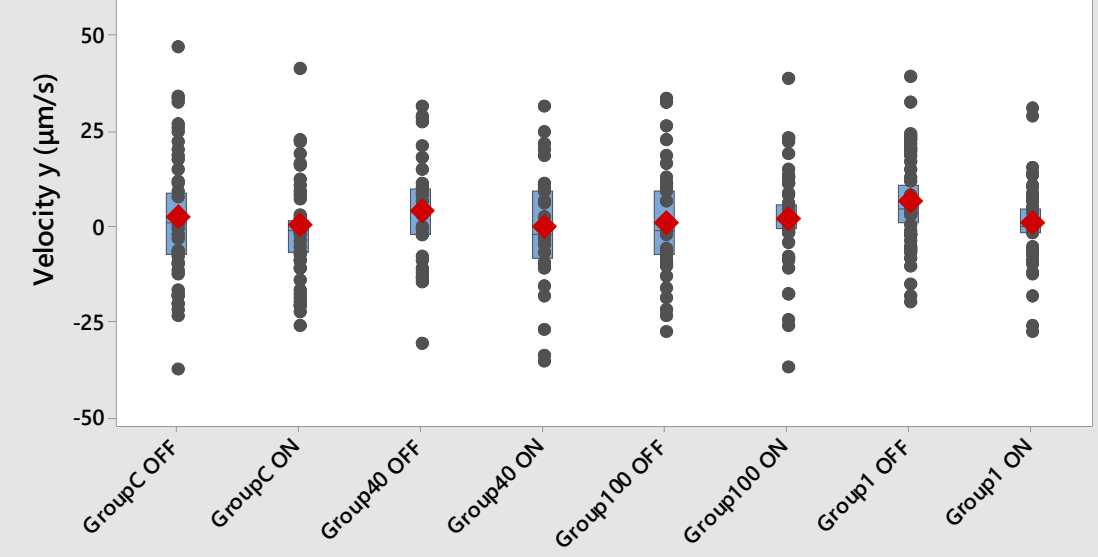



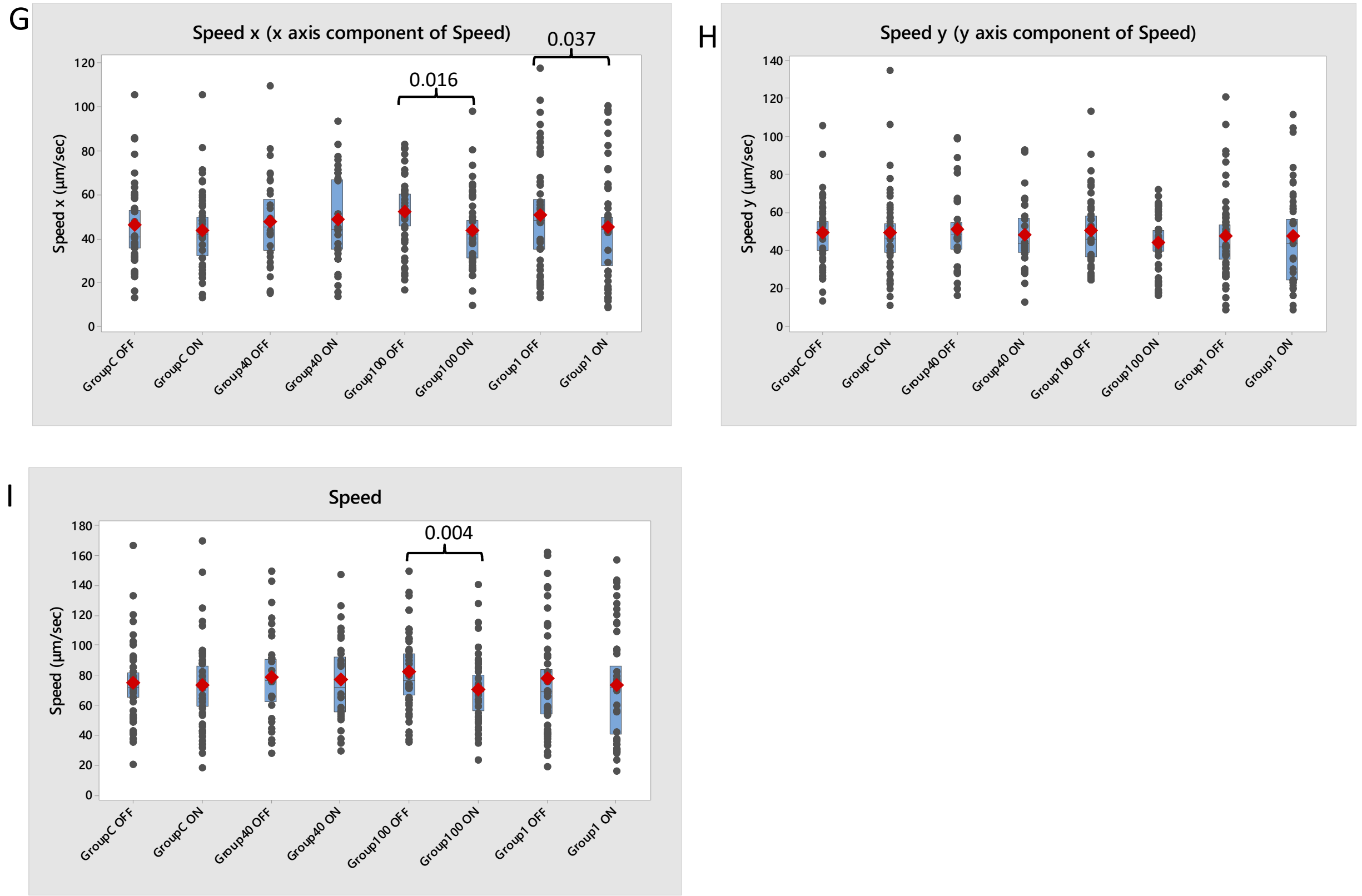
Figure 6 (continued)

\section{Path Features (J-M)}
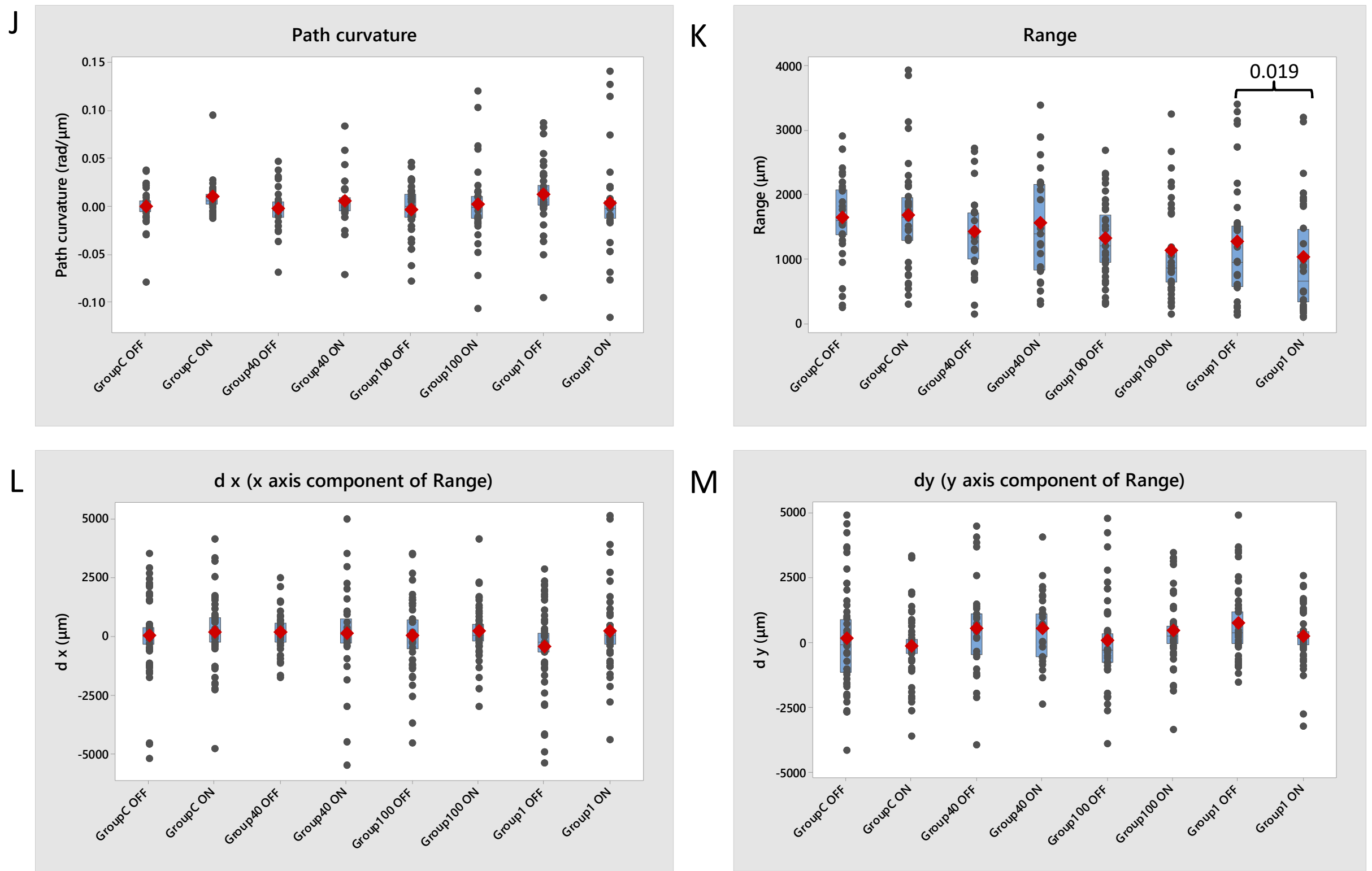
Fig. 6: Locomotion features and their metrics, as they have been quantified for all four groups of worms tested (Group C: control animals, fed on plain food source E. coli OP50, n=29; Group 1: fed on E. coli OP50 mixed with $1 \mu$ m-diameter paramagnetic particles, $\mathrm{n}=33$; Group 100: fed on E. coli OP50 mixed with $100 \mathrm{~nm}$-diameter iron core paramagnetic particles, n=38; and Group 40: fed on E. coli OP50 mixed with $40 \mathrm{~nm}$-diameter iron core paramagnetic particles, $n=42$ ) in the absence (OFF state) or in the presence (ON state) of external magnetic field. A-B: Posture features, C-I: Motion features, J-M: Path features. For each group tested worms were tracked in 3 different experimental days. Grey dots represent individual worms; red diamonds represent the mean; blue boxes indicate the median confidence interval box, with a middle line indicating the median. Dashed lines show comparisons between worms of different groups in the absence of magnetic field (OFF state). Continuous lines show comparisons between the ON and OFF state of worms of the same group. All $p$-values given are calculated by the Wilcoxon Signed Rank test with confidence interval set at $95 \%$, and any difference was considered statistically significant when $p \leq 0.05$. The $p$-values for all comparisons are given in Tables S1 and S2. 
Supplementary Figures and Tables 


\section{Figure S1}

A

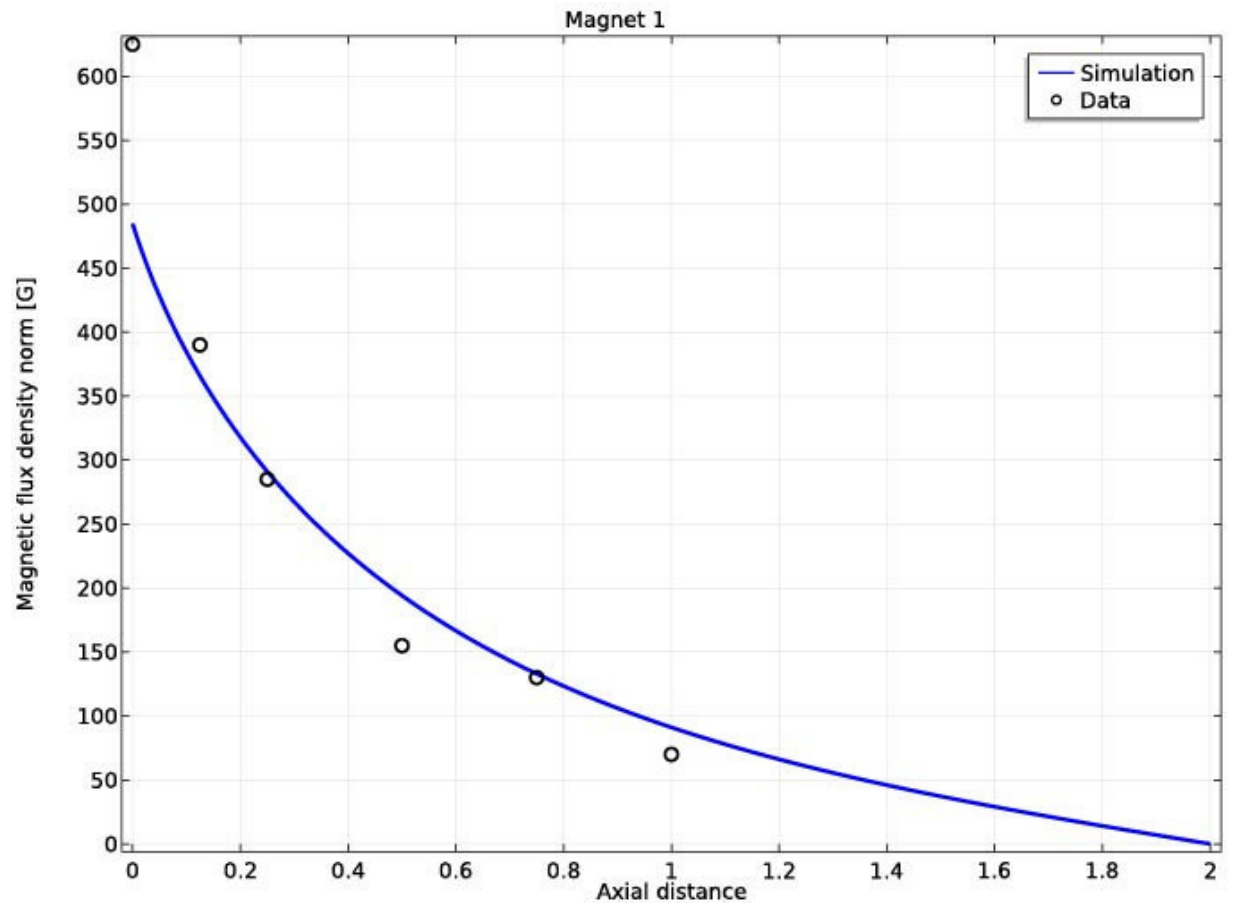

B

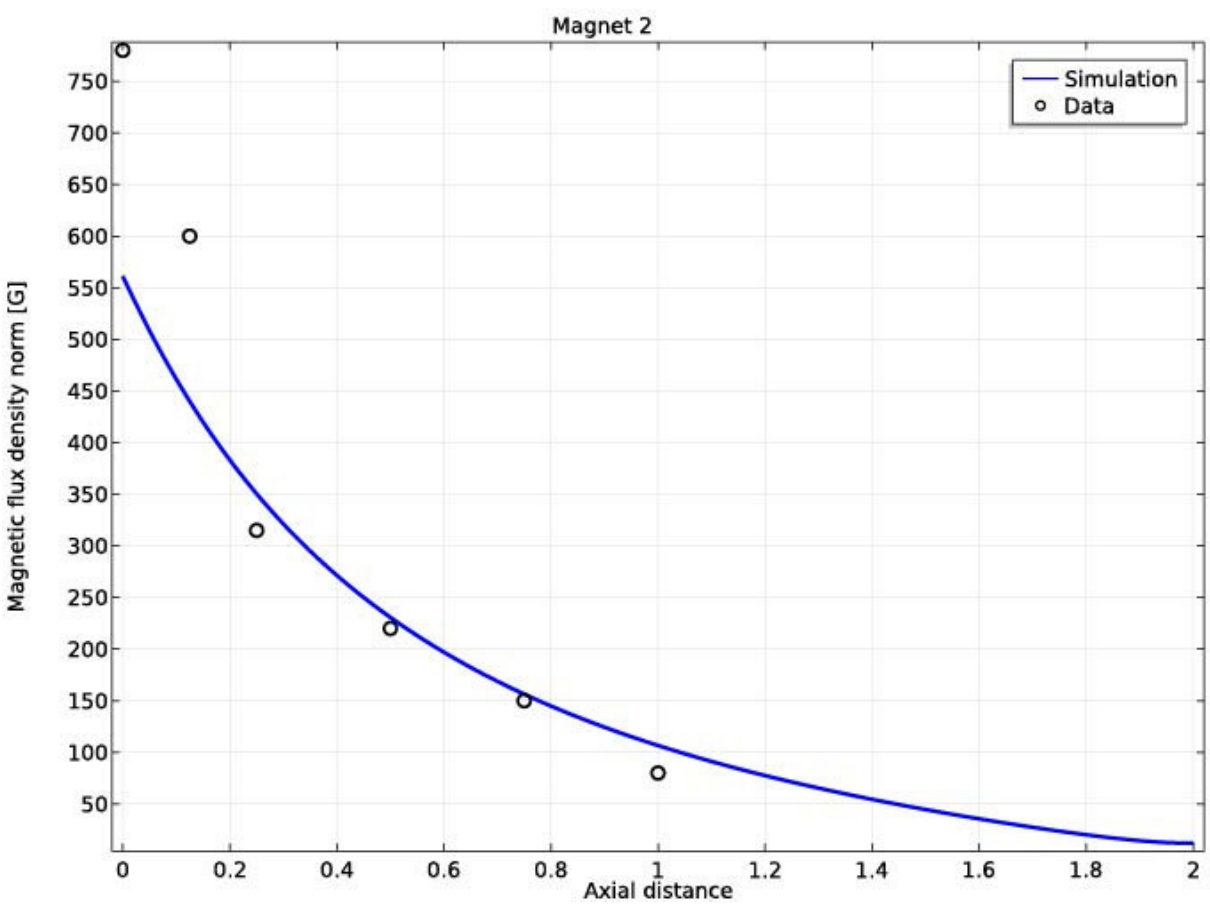

Fig. S1: Calibration of the parameters in COMSOL Multiphysics simulations to match the available data for magnet 1 (A) and magnet 2 (B) that are used in the experiments. 


\section{Figure S2}

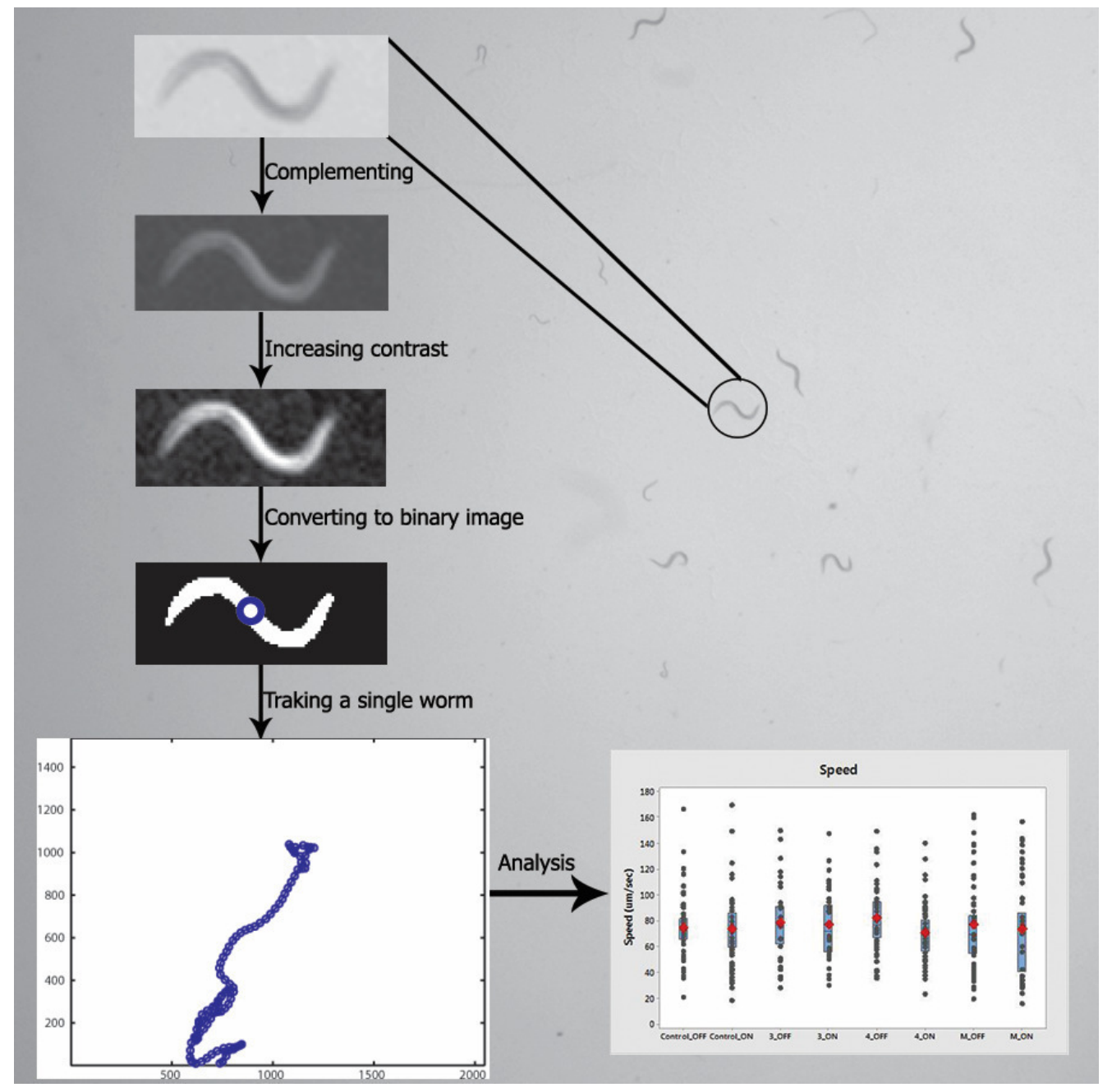

Fig. S2: An overview of the steps that are taken to analyze the locomotion of the worms. Top to bottom: A worm is selected in the first frame to be tracked. Next, several image enhancements are performed on the subframe that is created around the selected worm. Next, the grayscale image is converted to a binary image and postprocessing, e.g. finding the centroid (blue circle) of the worm, is performed. All these steps are conducted for the whole movie that is recorded. Finally, the data collected from all experiments are compared. 

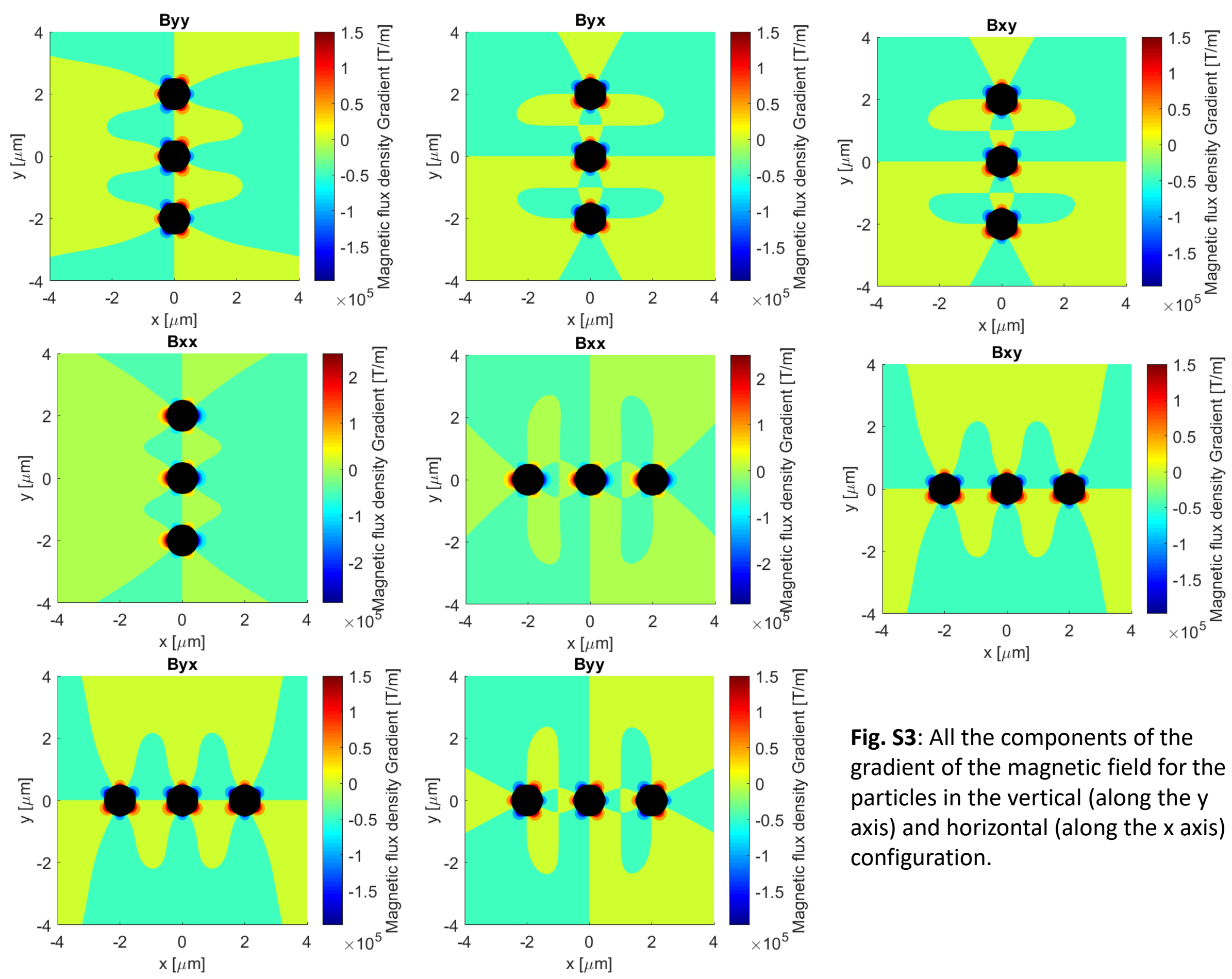

Fig. S3: All the components of the gradient of the magnetic field for the particles in the vertical (along the $y$ axis) and horizontal (along the $x$ axis) configuration. 


\section{Figure S4}

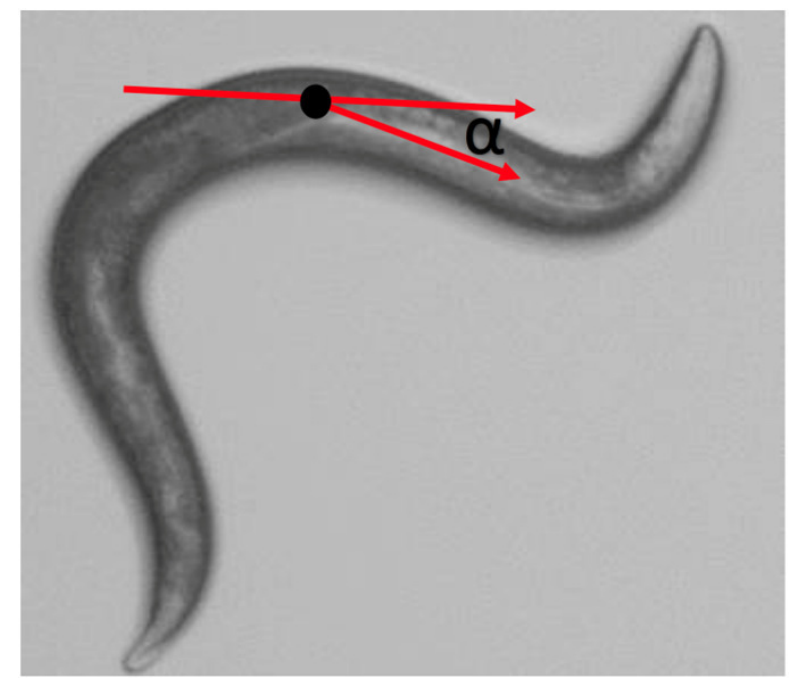

Fig. S4: The supplementary angle $(\alpha)$ is the difference in tangent angles at each skeleton point. 


\begin{tabular}{lcccc}
\hline \multicolumn{1}{c}{ Metric } & Group C OFF/ON & Group 1 OFF/ON & Group 100 OFF/ON & Group 40 OFF/ON \\
\hline Total bends & 0.645 & 0.645 & 0.644 & 0.540 \\
\hline Bend count & 0.052 & 0.093 & $\mathbf{0 . 0 3 1}$ & 0.764 \\
\hline Forward/backward ratio & 0.681 & 0.193 & $\mathbf{0 . 0 1 0}$ & 0.747 \\
\hline Stay ratio & 0.967 & 0.336 & $\mathbf{0 . 0 0 7}$ & 0.706 \\
\hline Velocity x & 0.832 & 0.375 & 0.477 & 0.979 \\
\hline Velocity y & 0.599 & 0.066 & 0.514 & 0.375 \\
\hline Speed x & 0.431 & 0.037 & 0.016 & 0.936 \\
\hline Speed y & 0.235 & 0.066 & 0.093 & 0.435 \\
\hline Speed & 0.438 & 0.190 & $\mathbf{0 . 0 0 4}$ & 0.554 \\
\hline Path curvature & 0.120 & 0.059 & 0.543 & 0.225 \\
Range & 0.773 & $\mathbf{0 . 0 1 9}$ & 0.104 & 0.706 \\
\hline D x & 0.960 & 0.280 & 0.499 & 0.914 \\
\hline D y & 0.626 & 0.250 & 0.217 & 0.920 \\
\hline
\end{tabular}

Table S1: The magnetic field effect on the locomotion of worms with internalized nanoparticles. $p$-values of Wilcoxon Signed Rank test for all comparisons between OFF and ON state of each group studied. With bold are highlighted the statistically significant differences, with $p$-value $\leq 0.05$. 


\begin{tabular}{lcccc}
\hline \multicolumn{1}{c}{ Metric } & Kruskal-Wallis & Group C vs Group 1 & Group C vs Group 100 & Group C vs Group 40 \\
\hline Total bends & 0.649 & & & \\
\hline Bend count & 0.237 & & & \\
\hline Forward/backward ratio & 0.234 & & 0.064 \\
\hline Stay ratio & $\mathbf{0 . 0 4 5}$ & $\mathbf{0 . 0 0 5}$ & \\
\hline Velocity x & 0.729 & & \\
\hline Velocity y & 0.321 & & \\
Speed x & 0.498 & & \\
\hline Speed y & 0.804 & & \\
Speed & 0.661 & & \\
\hline Path curvature & 0.096 & & \\
\hline Range & 0.115 & & \\
\hline D x & 0.649 & & \\
\hline D y & 0.188 & & \\
\hline
\end{tabular}

Table S2: The particle effect on the locomotion of worms with internalized nanoparticles. In the second column are given the $p$-values of Kruskal-Wallis test used to compare all groups of worms studied during their OFF state. In the last three columns are given the $p$-values of the Wilcoxon Signed Rank test used to compare Group C with each of the other three particle-containing groups. The Wilcoxon Signed Rank test was run only when Kruskal-Wallis test gave a $p$-value $\leq 0.05$. With bold are highlighted the statistically significant differences, with $p$-value $\leq 0.05$. 
A

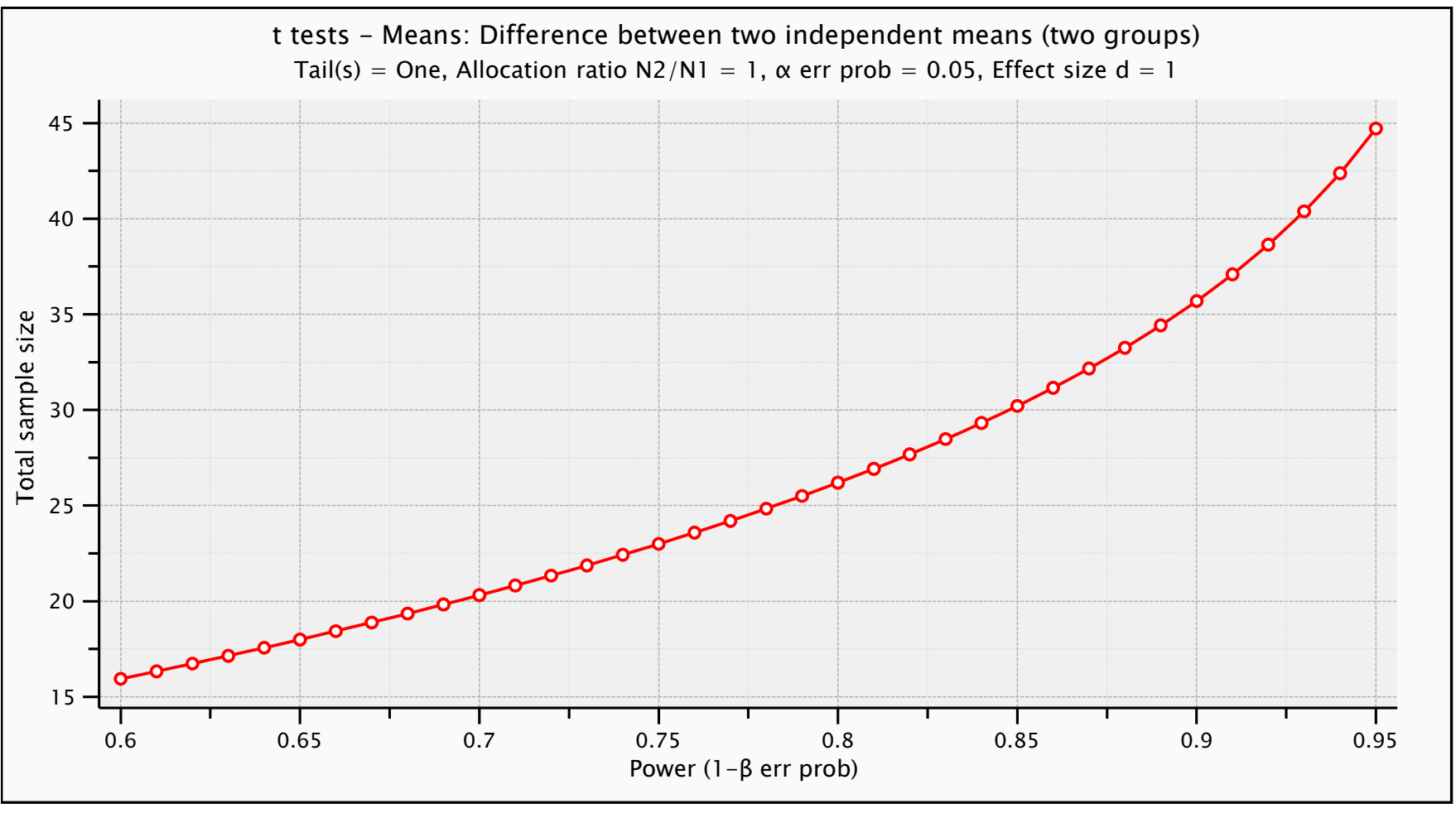

B

Analysis: A priori: Compute required sample size

Input: Tail(s) $=$ One

Effect size $\mathrm{d}=1.0000000$

$\alpha$ err prob $=0.05$

Power $(1-\beta$ err prob) $=0.95$

Allocation ratio $\mathrm{N} 2 / \mathrm{N} 1=1$

Output: Noncentrality parameter $\delta=3.391165$

Critical $t=1.680230$

$\mathrm{Df}=44$

Sample size group $1=23$

Sample size group $2=23$

Total sample size $=46$

Actual power $=0.954817$

Fig. S5: Power analysis for locomotion dynamics experiments. G*Power open source software was used as described in Methods. A. Plot that shows the probability of detecting a real effect with regard to sample size; $B$. Table showing the protocol followed for the power analysis. The mean of group1 was set to 0.5 and the mean of group2 was set to 0.4 , with SD within each group $\sigma=0.1$. 



\title{
Caenorhabditis elegans locomotion is affected by internalized paramagnetic nanoparticles in the presence of magnetic field
}

\author{
Eleni Gourgou ${ }^{1,2}$, Yang Zhang ${ }^{1,3}$, Ehsan Mirzakhalilii', Bogdan Epureanu ${ }^{1,+}$
}

1. Mechanical Engineering, University of Michigan, Ann Arbor, MI, United States

2. Internal Medicine, Division of Geriatrics, Medical School, University of Michigan, Ann Arbor, MI, United States

3. Computer and Electronic Engineering, UM-SJTU Joint Institution, Shanghai JiaoTong University, Shanghai, China

+ corresponding author: Bogdan Epureanu, epureanu@umich.edu

\section{Supplementary Information}

The features of the magnetic field (MF) presented here are independent of the magnetic particles and depend only on the properties of the electromagnets and the geometry of the setup. However, the forces that are exerted on the particles depend also on the properties of the particle. We have used several different magnetic particles in our experiments. The numerical simulations and analysis are performed only on the largest magnetic particles since such analysis provide us with the largest forces that can be created because of the external MF. Moreover, the characteristics of Dynabeads are more accessible (Fonnum et al., 2005) compared to the rest of the particles used in our experiments.

The forces on a magnetic particle in an external MF can be characterized as (Shevkoplyas et al., 2007):

$$
\mathbf{F}=\rho V \nabla\left(\mathbf{M}_{0} \cdot \mathbf{B}\right)+\frac{V \chi_{\text {bead }}}{\mu_{0}}(\mathbf{B} . \nabla) \mathbf{B},
$$

where $\rho, V, M_{0}, \chi_{\text {bead }}$ are the density, volume, initial magnetization, and initial magnetic susceptibility of the particles, respectively. $\mathbf{F}$ is the force acting on the particle, $\mathbf{B}$ is the external MF, and $\mu_{0}$ is the permeability of vacuum. 
Equation (1) is used with the properties of the magnetic particles (Fonnum et al., 2005) and the simulation results from COMSOL Multiphysics for the external MF to calculate the forces acting on the magnetic particles. Figure 2 shows that the magnitude of the force at all locations on the plate, which, as expected, can be observed to be larger near the electromagnets where the MF and its gradient are larger.

The magnetization of the particles creates local modifications to the MF compared to the externally applied MF. From classical physics, the MF around a magnetic dipole can be expressed as:

$$
\mathbf{B}(\mathbf{r})=\frac{\mu_{0}}{4 \pi}\left(\frac{3 \mathbf{r}(\mathbf{M} \cdot \mathbf{r})}{r^{5}}-\frac{\mathbf{M}}{r^{3}}\right),
$$

where $\mathbf{r}$ is position vector relative to the particle at which the MF is calculated and $\mathbf{M}$ is the magnetic moment of the particle that can be calculated as (Shevkoplyas et al., 2007):

$$
\mathbf{M}=\mathbf{M}_{0}+\frac{\chi_{\text {bead }}}{\rho} \frac{\mathbf{B}_{\text {external }}}{\mu_{0}}
$$

Equation (3) is used with the properties of the largest particles in our experiments (Fonnum et al., 2005) along with the external MF ( $\mathbf{B}_{\text {external }}$ ) created by the electromagnets obtained from COMSOL Multiphysics to determine the magnetic moments of the particles in the presence of the external MF. Next Eq. (2) is used to calculate the MF around the particles when the magnetic moment is known. Figure 3 shows the magnitude of the MF flux for vertical and horizontal configuration of three magnetic particles using Eqs. (2) and (3) in MATLAB. The MF is strong close to the particles for both configurations, and decays rapidly with distance from the particles.

The MF shown in Figure 3 can also be used to calculate the gradient of the MF around the particles. Figure 3 show the gradient of the magnetic of for both horizontal and vertical configuration. Again, the gradient of the MF is strongest near the particles and decays rapidly with distance from the particles.

Finally, the forces between two magnetic particles can be found as (3):

$$
\mathbf{F}_{12}=-\frac{3 \mu_{0} M^{2}}{4 \pi r_{12}{ }^{4}}\left(-\left(\mathbf{n}_{1} \cdot \mathbf{n}_{2}\right) \mathbf{t}_{12}-\left(\mathbf{n}_{2} \cdot \mathbf{t}_{12}\right) \mathbf{n}_{1}-\left(\mathbf{n}_{1} \cdot \mathbf{t}_{12}\right) \mathbf{n}_{2}+5\left(\mathbf{n}_{1} \cdot \mathbf{t}_{12}\right)\left(\mathbf{n}_{2} \cdot \mathbf{t}_{12}\right) \mathbf{t}_{12}\right)
$$

where $\mathbf{F}_{12}$ is the force between the two particles, $r_{12}$ is the distance between the two particles, $\mathbf{t}_{12}$ is the unit vector that connects the two particles, $\mathbf{n}_{1}$ and $\mathbf{n}_{2}$ are the direction of the magnetic moment for particle 1 and 2 , and $M$ is the magnitude of the magnetic moment of the two 
particles. Here, we have assumed that the distance between the two particles is small leading to the same magnetic moment for both particles based on Eq. (3). Figure 3E shows how the force between two particles changes for both vertical and horizontal direction. The force between the particles in the horizontal direction is repulsive while the force between the particles in the vertical direction is attractive. The attractive forces between the particles cause them to form chain-like structures when they are not interrupted by the medium in which the particles are located (Mirzakhalili et al., 2017; Nakata et al., 2008).

\section{Comparison of the MF/gradient MF between particles}

We compare the MF and gradient of MF between the particles based on their size. The iron mass of an ideal spherical particle scales with $d^{3}$, where $d$ is the diameter of the particle. Hence, for constant density, smaller particles have smaller magnetic mass by a factor of $d^{-3}$. However, according to Eq. 2 in the Supplementary Information, the MF around a particle scales with $r^{-3}$, where $r$ is the distance from the center of the particle. Therefore, the MF on the surface of a particle changes with its size. Smaller particles have less magnetic material, so their MF in the proximity of the particle is smaller. In addition, the gradient of MF scales with $r^{-4}$ . Hence, since the mass of the magnetic core scales with $d^{-3}$, the gradient of MF on the surface of the particle scales with $d$. Therefore, smaller particles will have larger gradient of MF compared to larger particles in their proximity.

\section{References}

Fonnum, G., Johansson, C., Molteberg, A., Mørup, S. \& Aksnes, E. 2005. Characterisation of Dynabeads ${ }^{\circledR}$ by magnetization measurements and Mössbauer spectroscopy. Journal of Magnetism and Magnetic Materials, 293, 41-47.

Mirzakhalili, E., Nam, W. \& Epureanu, B. I. 2017. Reduced-order models for the dynamics of superparamagnetic nanoparticles interacting with cargoes transported by kinesins. Nonlinear Dynamics, 90, 425-442.

Nakata, K., Hu, Y., Uzun, O., Bakr, O. \& Stellacci, F. 2008. Chains of Superparamagnetic Nanoparticles. Advanced Materials, 20, 4294-4299. 

not certified by peer review) is the author/funder. All rights reserved. No reuse allowed without permission.

Shevkoplyas, S. S., Siegel, A. C., Westervelt, R. M., Prentiss, M. G. \& Whitesides, G. M. 2007. The force acting on a superparamagnetic bead due to an applied magnetic field. Lab Chip, 7, 1294-302. 